\title{
Thickness, porosity, and permeability prediction: comparative studies and application of the geostatistical modeling in an Oil field
}

\author{
Shan Zhao*, Yang Zhou, Mengyuan Wang, Xiaying Xin and Fang Chen
}

\begin{abstract}
Background: In this study, we applied the geostatistical modeling to analyze an oil field. The reservoir properties, thickness, porosity and permeability, were studied. Data analysis tools, such as histogram, scatter plot, variogram and cross variogram modeling, were employed to capture the interpretable spatial structure and provide the desired input parameters for further estimation. SK (simple kriging), OK (ordinary kriging), Sgism (Sequential Gaussian Simulation), SC (simple cokriging), OC (ordinary cokriging) and MM2 (Markov model 2) methods were applied to estimate reservoir properties. Estimation difference maps were generated to compare the results of each method, providing more straightforward realizations in a visual way.

Results: For thickness, results indicated that anisotropic variogram could provide better interpretations for the spatial relationships than isotropic variogram. Both SK and OK could provide better estimates. In comparison to the conventional estimation techniques, the simulation method could well reflect the reservoir's intrinsical characteristics in terms of the associated extreme values. OOIP (Original Oil In Place) was calculated later with the parameters attained before, including thickness and porosity. Estimation difference maps showed that there was no obvious difference in SK vs. OK and SC vs. OC for the study of permeability. However, OC was slightly different from OK, and there were significant discrepancies between the estimates of OC and MM2 at the unsampled locations. In addition, OC estimates were closest to the sample data of permeability with the minimum variance.
\end{abstract}

Conclusions: Geostatistical modeling is an effective way for thickness, porosity, and permeability prediction.

Keywords: Geostatistics; Variogram; Kriging; Thickness; Porosity; Permeability

\section{Background}

Maps and mapmaking are integral parts of reservoir characterization. A map is a numerical model of an attribute's (e.g., porosity, permeability, thickness, structure) spatial distribution (Malvić and Jović 2012; Huysmans and Dassargues 2013). However, mapping an attribute is rarely the goal; rather, a map is used to make a prediction about the reservoir. To paraphrase Andre Journel of Stanford University, "A map is a poor model of reality if it does not depict characteristics of the real spatial distribution of those attributes that most affect how the reservoir responds".

\footnotetext{
* Correspondence: shan.zhao2010@gmail.com

Faculty of Engineering and Applied Science, University of Regina, Regina, Saskatchewan S4S 0A2, Canada
}

The enormous up-front investments for developing heterogeneous fields and the desire to increase ultimate recovery have spurred oil companies to use innovative reservoir characterization techniques (Habibnia and Momeni 2012; Abdideh and Mahmoudi 2013). Geostatistics is one of many new technologies often incorporated into the process (Cressie and Hawkins 1980; Bueno et al. 2011). For more than a decade, geostatistical techniques, especially when incorporating 3-D seismic data, have been an accepted technology to characterize petroleum reservoirs (Qi et al. 2007; Abdideh and Bargahi 2012; Esmaeilzadeh et al. 2013; Fegh et al. 2013).

Geostatistical application necessitates and facilitates cooperation between geoscientists and reservoir engineers, allowing each discipline to contribute fully. This is quite different from the past, because the mathematical formalization was often left to the reservoir engineer. 
Thus, part of the geostatistical philosophy is to ensure that geologic reality does not get lost during reservoir model building (Nava et al. 2010; Chen et al. 2011).

Geostatistics attempts to improve predictions by developing a different type of quantitative model. The goal is to construct a more realistic model of reservoir heterogeneity using methods that do not average important reservoir properties. Like the traditional deterministic approach, it preserves indisputable "hard" data where they are known and interpretative "soft" data where they are informative (Wilson et al. 2011).

However, unlike the deterministic approach, geostatistics provides numerous plausible results (Zarei et al. 2011). The degree to which the various models differ is a reflection of the unknown or a measurement of the "uncertainty". Some outcomes may challenge prevailing geologic wisdom and will almost certainly provide a range of economic scenarios, from optimistic to pessimistic. Having more than one result to analyze changes the paradigm of traditional reservoir analysis and may require multiple reservoir flow simulations (Schmidt and Schröder 2011; Soleymani and Riahi 2012). However, the benefits outweigh the additional time and cost.

The Stanford Geostatistical Modeling Software (SGeMS) is an open-source computer package for solving problems involving spatially related variables. It provides geostatistics practitioners with a user-friendly interface, an interactive 3-D visualization, and a wide selection of algorithms (Kelsall and Wakefield 2002). This website serves as a companion to the book Applied Geostatistics with SGeMS that provides a step-by-step guide to using SGeMS algorithms. We recommend getting the book to get the underlying theory, demonstrations of their implementation, discussion of potential limitations, and help about the choice of one algorithm over another.

Users can perform complex tasks using the embedded Python scripting language, and new algorithms can be developed using the SGeMS plug-in mechanism. SGeMS is the first software to provide algorithms for multiplepoint statistics. The SGeMS package provides a versatile toolkit for Earth Sciences graduates and researchers, as well as practitioners of environmental, mining and petroleum engineering.

SGeMS provides a (fairly) comprehensive collection of geostatistical estimation and simulation algorithms and also provides a nice 3D visualization environment (Kelkar and Perez 2002; Remy 2004; Geoff 2007; Remy et al 2009). It provides a more limited selection of options for standard statistical data analysis and essentially no facilities for data management (subsetting data sets, etc.). So, you would probably want to run S-GeMS in tandem with some other data analysis \& management software, such as Excel.

In the following chapters of this study, we will use SGeMS to make estimation about an oilfiled step by step. The user selects in this panel which geostatistics tool to use and inputs the required parameters. The top part of that panel shows a list of available algorithms, e.g. kriging, sequential Gaussian simulation. When an algorithm from that list is selected, a form containing the corresponding input parameters appears below the tools list. One or multiple objects can be displayed in this panel, e.g. a Cartesian grid and a set of points, in an interactive 3D environment. Visualization options such as color-maps are also set in the Visualization Panel.

\section{Results and discussion}

This study started by loading data from a file named Flow Unit 5, a DAT-format data file containing thick, porosity and permeability data from about 68 wells in Flow Unit 5 Oil Field.

\section{Thickness}

\section{Simple kriging and ordinary kriging of isotropy}

Simple kriging Figure 1a shows the gross thickness map generated with isotropic simple kriging. Overall, the map is not that smooth. In the northwest corner, the conditioning data is undersampled with respect to the rest of the grid. The isotropic simple kriging variance map is shown in Figure 1b. The estimation variance is pretty high through the entire map. Small variance only exists in places where there are wells distributed. The histogram, for the simple kriging estimates, is shown in figure. Most of the data are distributed close to the centre, with some extreme values more than $10 \mathrm{ft}$. The maximum estimate value is $24.61 \mathrm{ft}$, while the maximum for the data is $27 \mathrm{ft}$. There is almost no improvement compared to the histogram of the conditioning data. 92 points are compared and plotted in the scatter plot of gross thickness data and isotropic simple kriging estimation data. The correlation coefficient is 2.082, indicating that isotropic SK tends to overestimate the estimates.

Ordinary kriging Figure 2a shows the gross thickness map generated with isotropic ordinary kriging. Overall, the map is not that smooth. The continuity is not that good even if it is a bit better than the map generated from simple kriging. In the northwest corner, the conditioning data is undersampled with respect to the rest of the grid. The isotropic ordinary kriging variance map is shown in Figure $2 \mathrm{~b}$. The estimation variance is pretty high through the entire map. Small variance only exists in places where there are wells distributed. The histogram, for the isotropic ordinary kriging estimates, is shown in figure. Most of the data are distributed close to the centre, with some extreme values more than $10 \mathrm{ft}$. The maximum estimate value is $25.036 \mathrm{ft}$, while the maximum for the data is $27 \mathrm{ft}$. There is almost no big improvement compared to the histogram of the conditioning data. 92 points are 


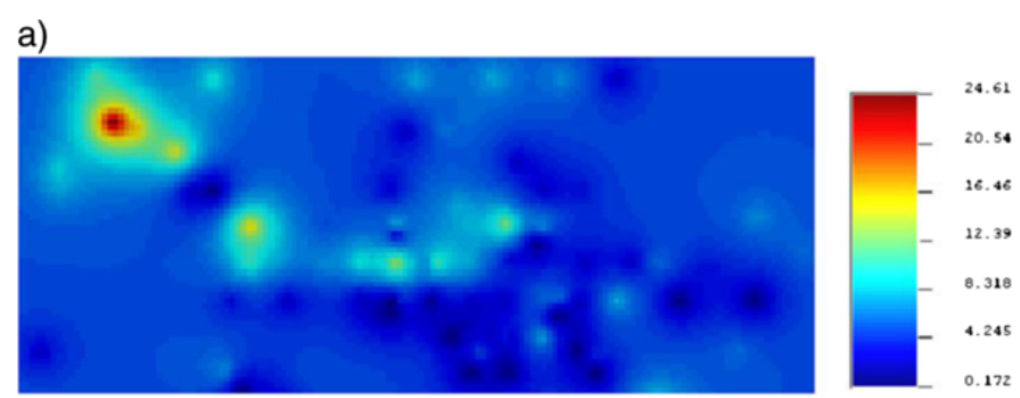

\section{b)}

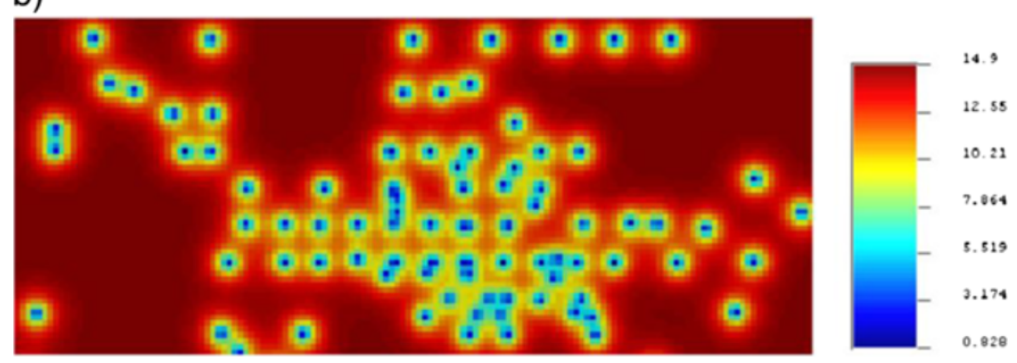

c)

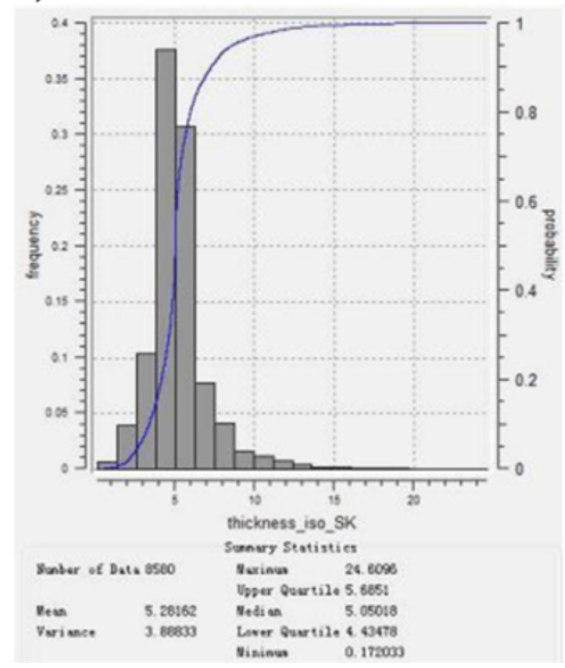

d)

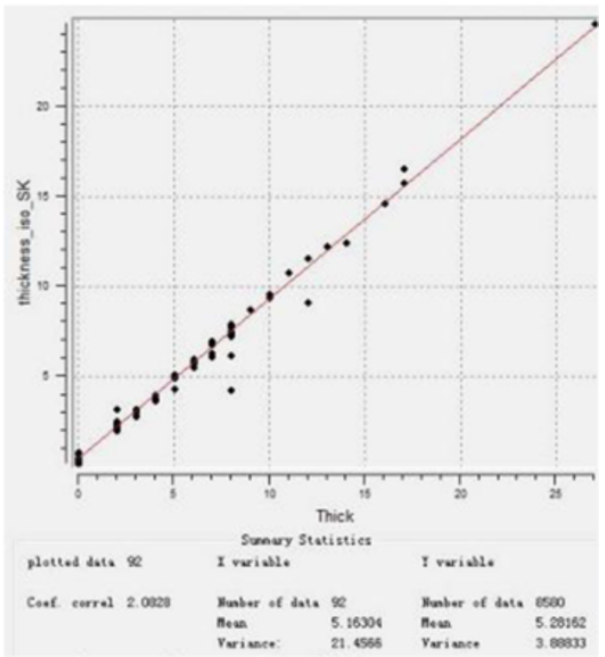

Figure 1 Thickness: a) Isotropic Simple Kriging; b) Isotropic Simple Kriging Variance; c) Histogram for Isotropic Simple Kriging; d) Compare with thickness and Isotropic simple kriging.

compared and plotted in the scatter plot of gross thickness data and isotropic ordinary kriging estimation data. The correlation coefficient is 1.384 , indicating that isotropic simple kriging tends to overestimate the estimates.

\section{Simple kriging and ordinary kriging of anisotropy}

Simple kriging Figure 3a shows the gross thickness map generated with simple kriging. Overall, the map has a smooth appearance that is typical of simple kriging. The good spatial continuity from east to west corresponds to the principal direction of the variogram. The north/ south trend, observed in a small area in the northwest corner of the map, is a result from the configuration of the conditioning data and the search neighborhood. In the northwest corner, the conditioning data is undersampled with respect to the rest of the grid. The simple kriging variance map is shown in Figure 3b. The estimation variance is small in gridblocks close to the conditioning data, and it becomes large in area far from the data. Near the conditioning data, the kriging variance becomes the nugget effect of the variogram. The histogram, for the simple kriging estimates, is shown in figure. The mean is 5.43 , the median is 4.88 and the mode is around 4, all of which are pretty close to the 




b)

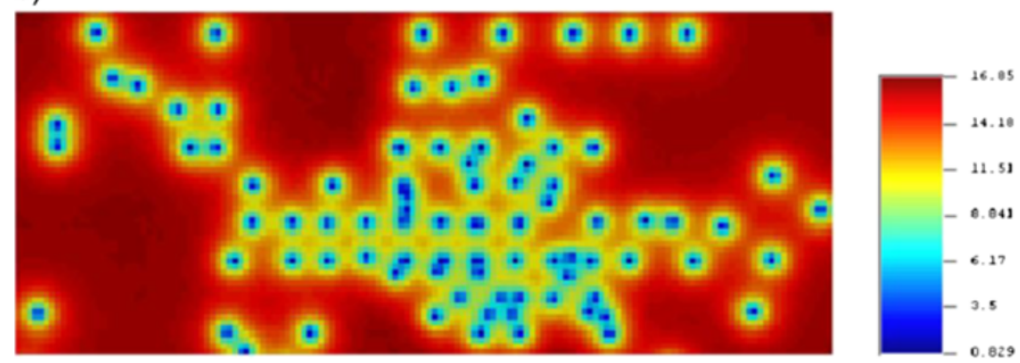

c)



d)

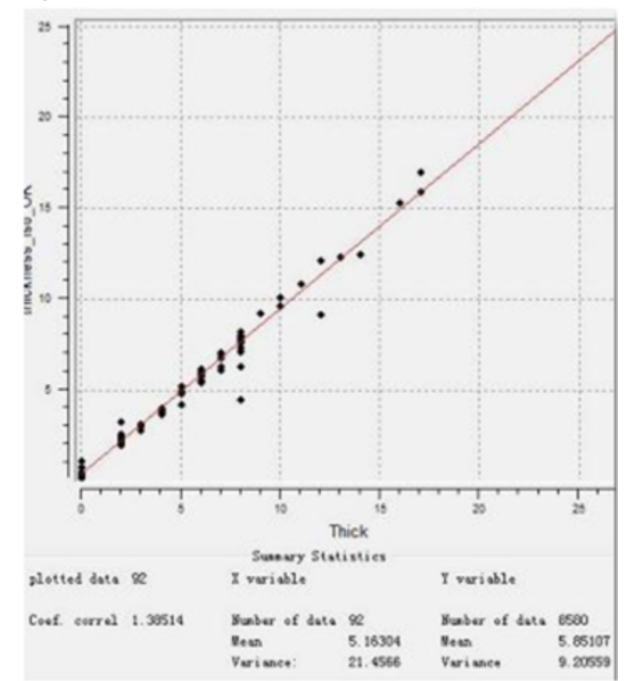

Figure 2 Thickness: a) Isotropic Ordinary Kriging; b) Isotropic Ordinary Kriging Variance; c) Histogram for Isotropic Ordinary Kriging; d) Compare with thickness and Isotropic Ordinary kriging.

conditioning data. The standard deviation of simple kriging estimates is $2.63 \mathrm{ft}$, which for conditioning data, it $4.53 \mathrm{ft}$. So it is likely that has a narrower spread than the conditioning data. In general, simple kriging does not closely reproduce the extreme values of a distribution with a gross thickness greater than $10 \mathrm{ft}$, observed in conditioning data. The maximum estimate value is $17.57 \mathrm{ft}$, while the maximum for the data is $27 \mathrm{ft}$. Simple kriging tends to make the data follow normal distribution, most of the data are distributed close to the mean value. 92 points are compared and plotted in the scatter plot of gross thickness data and simple kriging estimation data. Simple kriging provides good estimates even for values that are much larger than the mean of the data $(5.05 \mathrm{ft})$. All the plotted points fall approximately along the straight line, with correlation coefficient 0.891 , indicating high accuracy of the estimates.

Ordinary kriging Figure 4a shows the gross thickness map generated with ordinary kriging. Overall, the map has a more smooth appearance than that of simple kriging. Transition areas exist in ordinary kriging map, which 


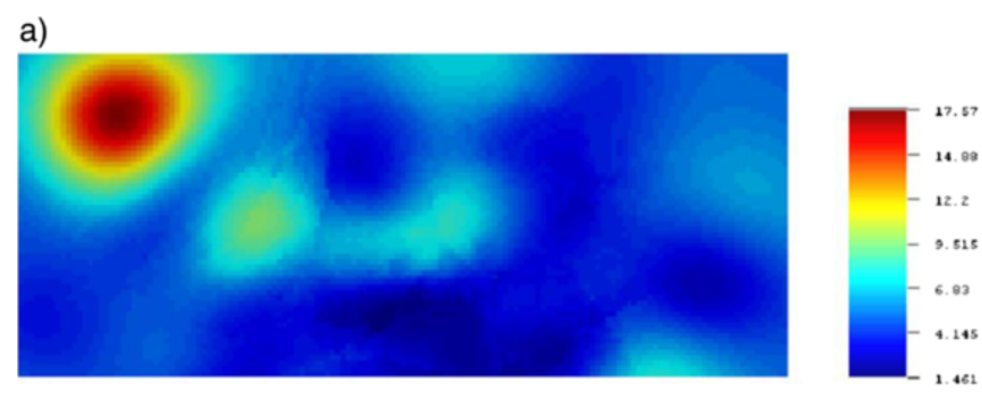

b)

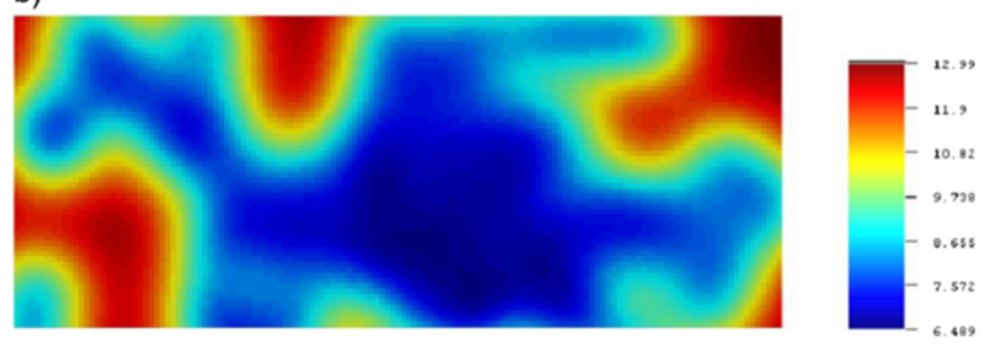

c)

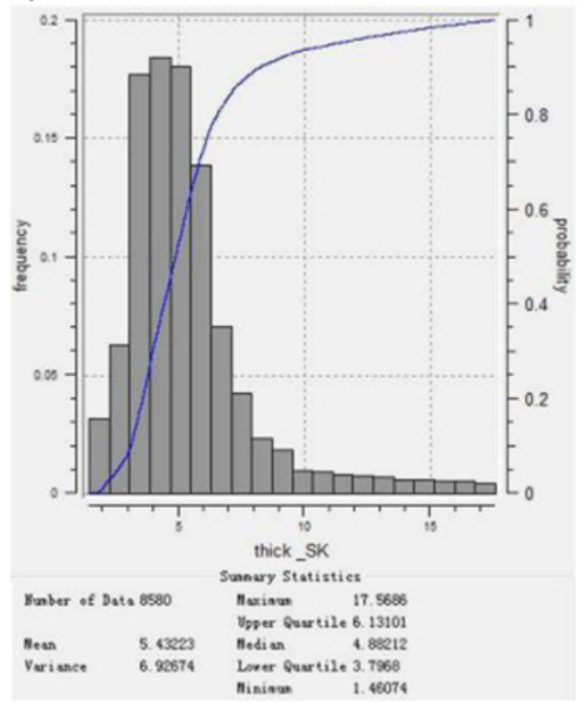

d)



Figure 3 Thickness: a) Anisotropic Simple Kriging; b) Anisotropic Simple Kriging Variance; c) Histogram for Anisotropic Simple Kriging; d) Compare with thickness and Anisotropic simple kriging.

represent gradual change among the subareas. The good spatial continuity from east to west corresponds to the principal direction of the variogram. The north/south trend, observed in a small area in the northwest corner of the map, is a result from the configuration of the conditioning data and the search neighborhood. In the northwest corner, the conditioning data id undersampled with respect to the rest of the grid. And this area tends to be larger than that of the simple kriging. Generally, the two maps are similar with each other. The ordinary kriging variance map is shown in Figure 4b, which is smoother than that of the simple kriging variance map. The blue area that represents small variance tends to be wider, which stands for a better estimation result compared to simple kriging. The estimation variance is small in gridblocks close to the conditioning data, and it becomes large in area far from the data. Near the conditioning data, the kriging variance becomes the nugget effect of the variogram. The histogram, for the ordinary kriging estimates, is shown in figure. The mean is 5.68 , the median is 4.89 and the mode is around 4, all of which are pretty close to the conditioning data. The standard deviation of ordinary kriging estimates is $2.98 \mathrm{ft}$, while for conditioning data, it is $4.53 \mathrm{ft}$. So it is likely that has a narrower spread 




b)

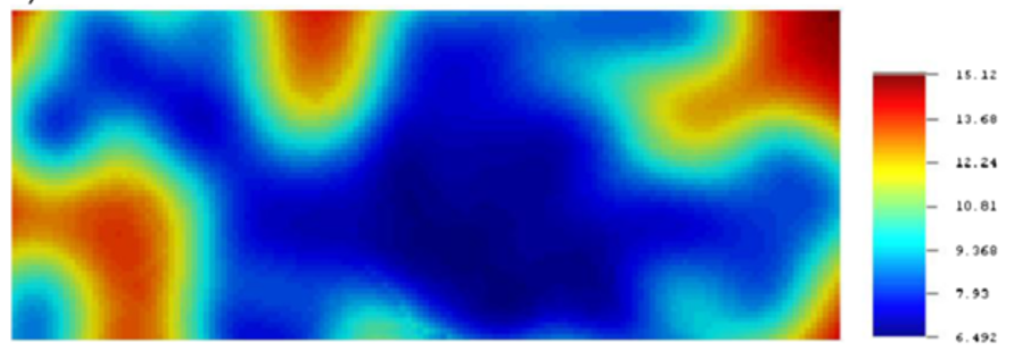

c)

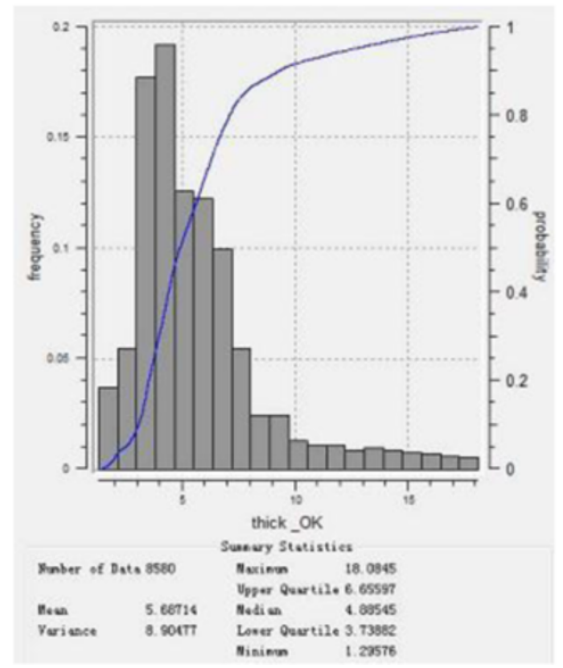

d)



Figure 4 Thickness: a) anisotropic ordinary kriging; b) anisotropic ordinary kriging variance; c) histogram for anisotropic ordinary kriging; d) compare with thickness and anisotropic ordinary kriging.

than the conditioning data. In general, simple kriging does not closely reproduce the extreme values of a distribution with a gross thickness greater than $10 \mathrm{ft}$, observed in conditioning data. The maximum estimate value is $18.08 \mathrm{ft}$, while the maximum for the data is $27 \mathrm{ft}$. Ordinary kriging tends to make the data follow normal distribution; most of the data distribute close to the mean value. 92 points are compared and plotted in the scatter plot of gross thickness data and ordinary kriging estimation data. Simple kriging provides good estimates even for values that are much larger than the mean of the data $(5.05 \mathrm{ft})$. All the plotted points fall approximately along the straight line, with correlation coefficient 0.825 ,though it is slightly smaller than the simple kriging.

\section{Comparison between isotropic and anisotropic variogram modeling}

From the thickness estimate of isotropic and anisotropic modeling, as shown in Figure 1 and Figure 2, it is obvious that anisotropic variogram is much smoother than the isotropic one. From the isotropic and anisotropic variance maps, the estimation variance is much lower for the anisotropic variogram than the isotropic one. This can further be demonstrated by the scatter plot of isotropic OK and anisotropic OK variance, where the 
isotropic variance is much larger than the anisotropic variance. All these illustrate that anisotropic estimation is more close to the conditioning data and is more accurate.

Through the above comparison between the isotropic modeling and anisotropic modeling, we draw a conclusion that anisotropic variogram can achieve better spatial structure capture more interpretable spatial relationship. In the following analysis of porosity and permeability properties, anisotropic variogram is mainly used to generate the spatial relationship of flow unit 5 .

\section{Porosity}

\section{Simple kriging}

Figure 5a shows the porosity map generated via simple kriging. Overall, the map has a smooth appearance. The good spatial continuity from middle to west corresponds to the principal direction of the variogram. The simple kriging variance map is shown in Figure 5b. The estimation variance could be as low as 0.6369 at where gridblocks are close to the sample data. However, in the northeast corner, the estimation variance could be as large as 64.28 due to the scarcity of sample data. For porosity data, the mean and median are 0.41 and 0.97 , respectively. They are very close to each other, indicating symmetry in the distribution. The coefficient of variation could be obtained via equation, and its value is 0.2735 , indicating a relatively small variation within the sample. The histogram is shown in Figure 5d. The mean is 0.67, the median is 0.39 and the variance is 25.5767 . Comparing with the histogram of sample data, the estimated values are generally a little smaller than the sample

a)



b)



c)

d)
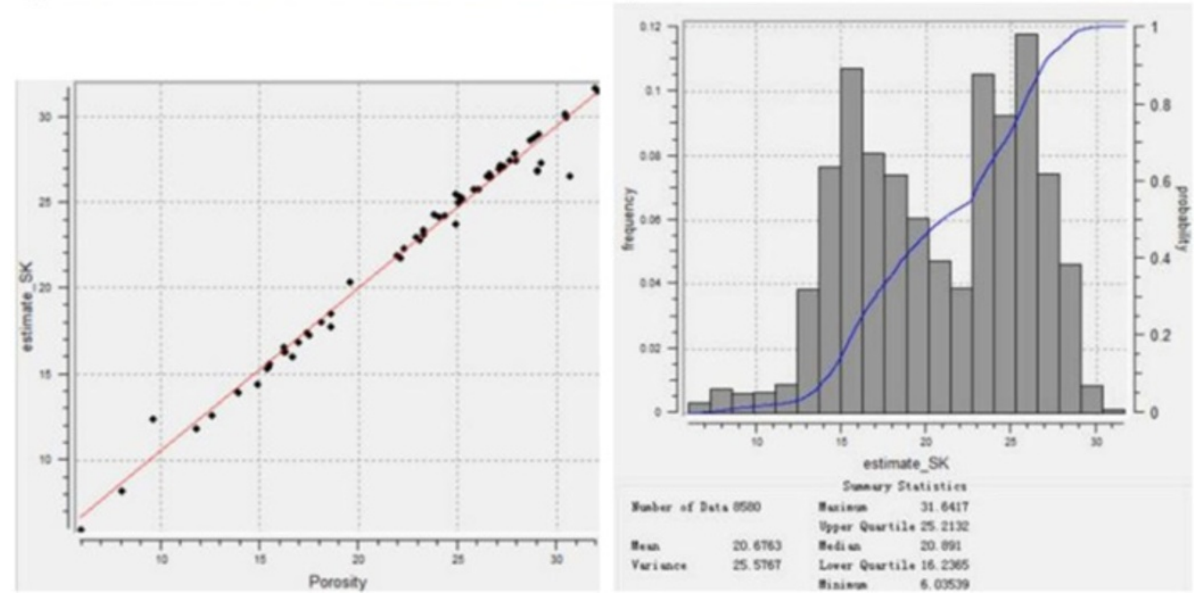

Figure 5 Porosity: a) simple kriging; b) simple kriging variance; c) coefficient of variation; d) histogram of sample data. 
data. However, the maximum and minimum values are quite close to that of samples respectively, indicating a relatively fine estimation. Moreover, the estimation variance is much smaller than the sample variance, which implies a relatively low variability in the estimated values. Similar conclusion can also be obtain via the coefficient of variation, which is 0.2446 .

\section{Ordinary kriging}

The porosity map from ordinary kriging is shown in Figure 6a. In general, this map is quite similar to the map generated via simple kriging. However, the appearance is much smoother. The variance map is shown in Figure $6 \mathrm{~b}$. The minimum variance is 0.637 , which is quite close to that from simple kriging. However, the maximum variance would be as large as 78.21 , which is noticeably larger than the maximum variance from simple kriging. The true value vs. the estimated value plot is shown in Figure 6c. All the points regularly spread around the $45^{\circ}$ line, indicating that the estimates generated via ordinary kriging can also match the sample data properly. The histogram is shown in Figure $6 \mathrm{~d}$. The mean is 0.7169 , the median is 0.4647 and the variance is 31.3116. Comparing with the histogram of sample data, the estimated values are also slightly smaller than the sample data. However, the maximum and minimum values are also quite close to that of samples respectively, indicating a relatively fine estimation. Comparing with the histogram of simple kriging's estimation, there is no significant difference in terms of their mean

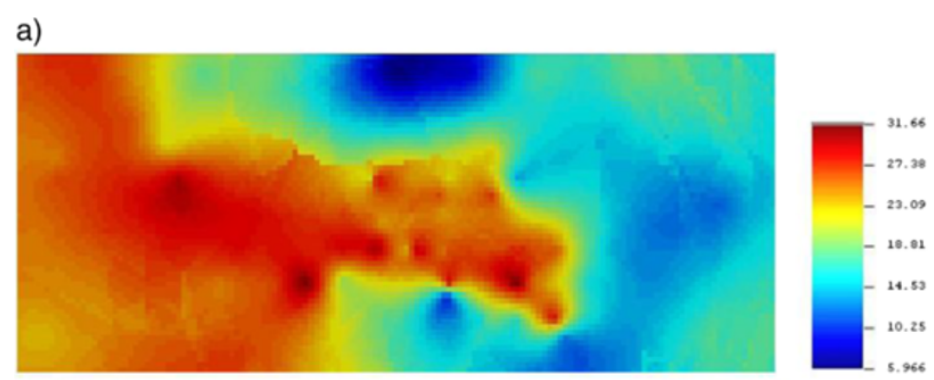

b)



c)

d)
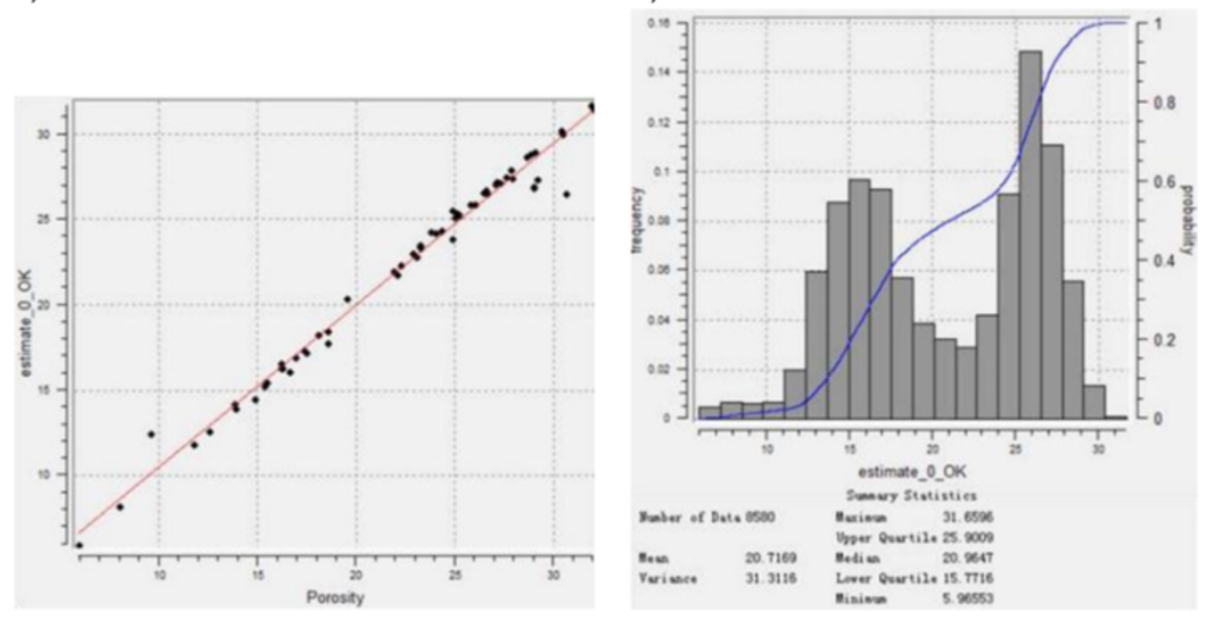

Figure 6 Porosity: a) ordianry kriging; b) ordinary kriging variance; c) coefficient of variation; d) histogram of sample data. 
values. However, the estimation variance from ordinary kriging method is much larger than that from simple kriging method.

\section{Sequential Gaussian simulation of porosity}

Next, we generate five realizations of porosity using sequential Gaussian simulation. The sequential Gaussian simulation will use a normal score transform to turn the porosity values at the wells into a set of values that perfectly follow a standard normal distribution (zero mean, unit standard deviation) and will then generate grids of simulated values whose univariate distribution is also standard normal. Simple kriging is to be applied since the spatially constant mean will be assumed to be zero. In addition, we assume that the variogram of the normal-score transformed data would look very similar to the variogram of the raw data scaled to a unit sill.
The simulation map is shown in Figure 7a. As can be seen from this figure, the distribution trend of the simulated porosity is quite similar to that from the simple kriging estimation as well as the ordinary kriging estimation. High porosity locations are spreading from middle to west. Noticeably, in the south-west corner, the simulated values of porosity are higher than either of the estimation results from the two kriging estimation methods. Figure $7 \mathrm{~b}$ shows the true value vs. the simulated value plot. All the points lie in the $45^{\circ}$ line, indicating a perfect match between true values and simulated values at sampled locations.

\section{Original Oil In Place (OOIP)}

OOIP is calculated with gross thickness and porosity maps generated with ordinary kriging OOIP in stock

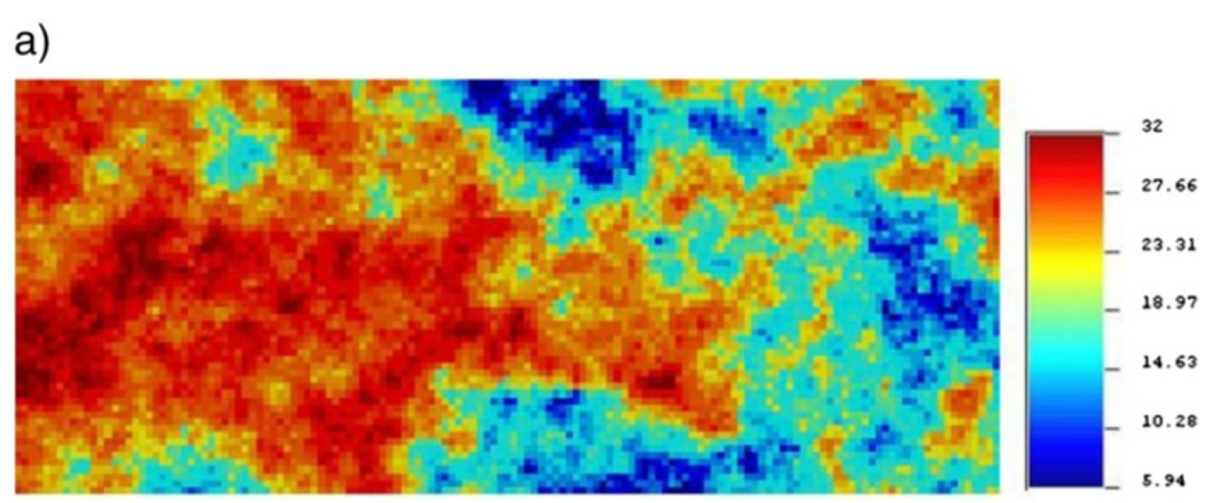

b)

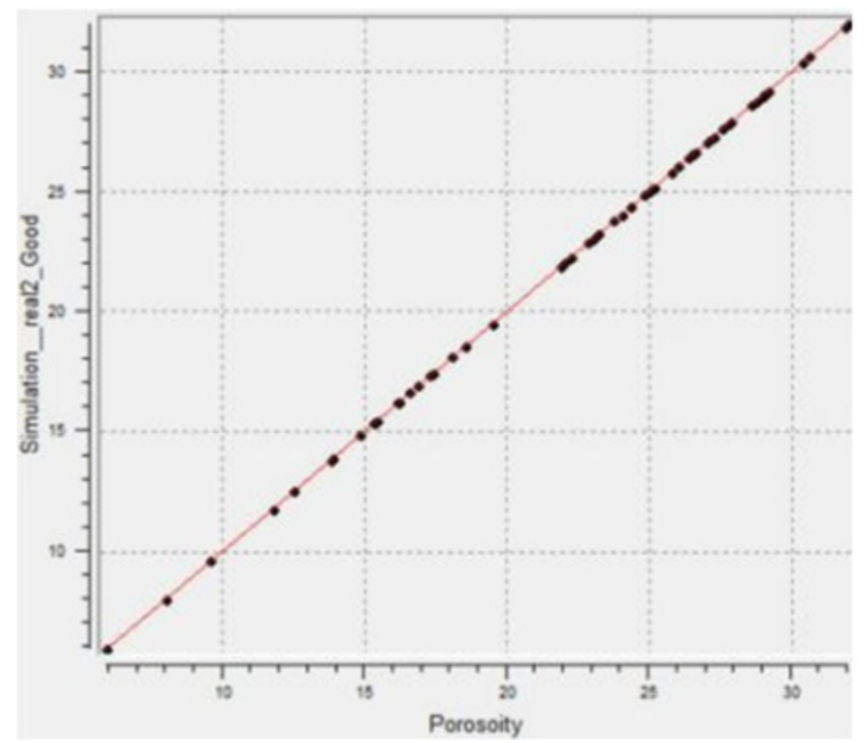

Figure 7 Porosity: a) plot of sequential gaussian simulation; b) true value vs. the simulated value plot. 
tank barrel (STB) for each gridblock in the map is given by:

$$
O O I P=\frac{A \times h \times n g \times(\phi / 100)(1-S w)}{5.615 \times B o}
$$

A $=$ the surface area of a block $(200 \times 200=40,000 \mathrm{ft} 2)$, $\mathrm{h}=$ gross thickness $(\mathrm{ft}), \mathrm{ng}=$ net to gross ratio, $\Phi=$ porosity (\%), $\mathrm{Sw}=$ the water saturation and Bo = formation volume factor (rest bbl/STB). So:

$$
\begin{aligned}
\text { OOIP } & =\frac{40000 \times 5.687 \times 0.7 \times(20.717 / 100)(1-20 \%)}{5.615 \times 1.2} \\
& \approx 3916.76
\end{aligned}
$$

\section{Permeability}

When considering the permeability, the values of $\mathrm{K}$ range from as low as 0.01 to $750 \mathrm{md}$, the majority of the values are at the lower end of the region. This type of histogram is rarely useful for characterizing a sample because the values are clustered at one end. One way to overcome this problem is to transform the sample data in some way so that some sample characteristics are evident from the histogram plot. The most commonly used approach for permeability values is the log transform. From analysis, the $\log \mathrm{k}$ distribution is much more symmetric than the permeability distribution. In addition, the log $\mathrm{k}$ and porosity histogram are remarkably similar. Both show similar trends with two peaks in the histogram plot, one of which is at the higher end of the values. Although this needs to be validated, such characteristic similarity way indicates a relationship between $\log \mathrm{k}$ and porosity.

\section{Simple kriging}

Figure 8 shows the permeability map generated via simple kriging. Overall, the map has a smooth appearance. The good spatial continuity from middle to west corresponds to the principal direction of the variogram. The simple kriging variance map is shown in Figure 8b variance of permeability. The estimation variance could be as low as 0.6369 at where gridblocks are close to the sample data. However, in the northeast corner, the estimation variance could be as large as 64.28 due to the scarcity of sample data. Then we use the Scatter Plot to compare the value of $\log \mathrm{k}$ (hard data) with SK value of logk. The plot shows that result of comparison is close to a $45^{\circ}$ slant, which means the value of estimation is really match the hard data very well.

\section{Ordinary kriging}

The permeability map from ordinary kriging is shown in Figure 9a. In general, this map is quite similar to the map generated via simple kriging. However, the appearance is much smoother. The variance map is shown in Figure 9b. The minimum variance is 0.637 , which is quite close to that from simple kriging. However, the maximum variance would be as large as 78.21, which is noticeably larger than the maximum variance from simple kriging.

\section{Simple cokriging}

Figure 10a shows the gross thickness map generated with simple cokriging. Overall, the map has a smooth appearance. The estimated distribution matches the original distribution well. The yellow and red areas represent the areas of which the permeability is relatively higher, while the blue areas stand for where the permeability is relatively lower. The good spatial continuity corresponds to the principal direction of the variogram. The small areas in the corner and bound of the map, is a result from the configuration of the conditioning data and the search neighborhood. In the northwest corner, the conditioning data is undersampled with respect to the rest of the grid. The simple cokriging variance map is shown in Figure 10b. The estimation variance is small in gridblocks close to the conditioning data, and it becomes large in area far from the data. Near the conditioning data, the kriging variance becomes the nugget effect of the variogram. The histogram, for the simple kriging estimates, is shown in figure. The mean is 1.90 , the median is 1.95 , both of which are a bit smaller than the conditioning data. The standard deviation of simple cokriging estimates is 0.78 ; while for conditioning data, it is 0.941 . So it is likely that has a narrower spread than the conditioning data. In general, Co-simple kriging tends to gather the data to the center, making the data follow normal distribution. That is, most of the data distributes close to the mean value. 55 points are compared and plotted in the scatter plot of gross log $\mathrm{k}$ data and simple cokriging estimation data. Simple kriging provides good estimates even for values that are a bit smaller than the mean of the data (1.90 to 2.13). All the plotted points fall approximately along the straight line, indicating high accuracy of the estimates.

\section{Ordinary cokriging}

Figure 11a shows the gross thickness map generated with ordinary cokriging. Overall, the map has a more smooth appearance than that of simple cokriging. Transition areas exist in ordinary cokriging map, which represent gradual change among the subareas. The estimated distribution matches the original distribution well. The yellow and red areas represent the areas of which the permeability is relatively higher, while the blue areas stand for where the permeability is relatively lower. The good spatial continuity corresponds to the principal direction of the variogram. The small areas in the corner and bound of the map, is a result from the configuration of the conditioning data and the search neighborhood. In the northwest corner, the 


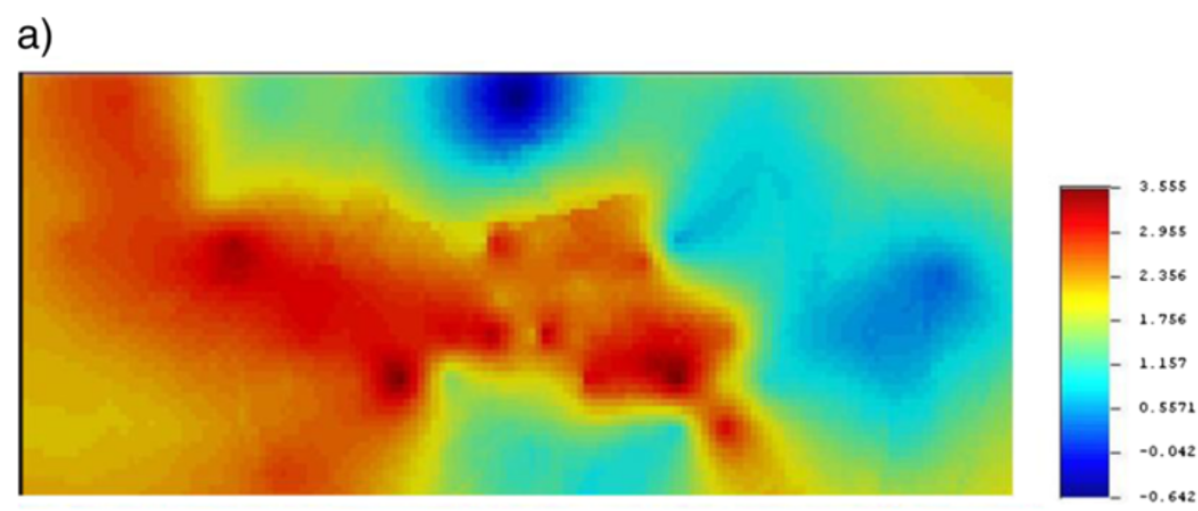

\section{b)}

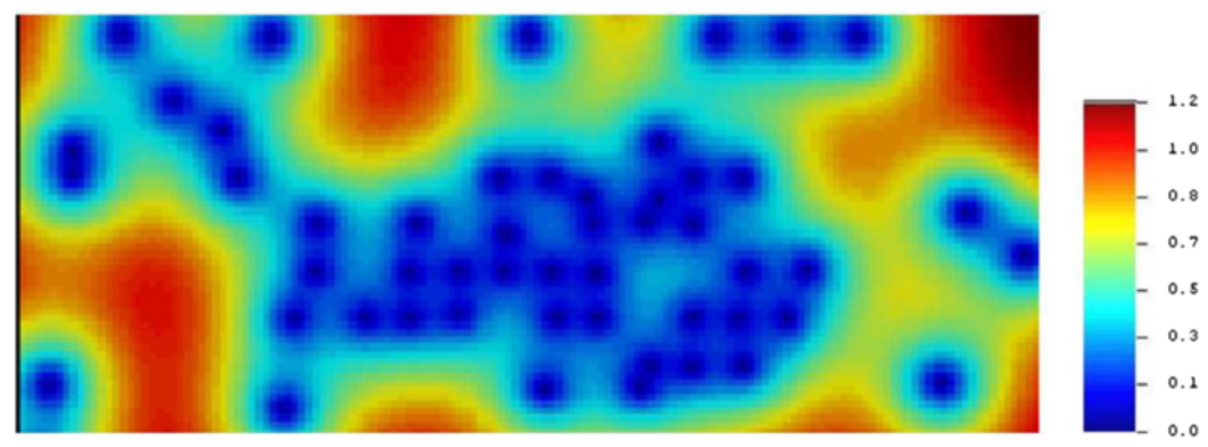

c)

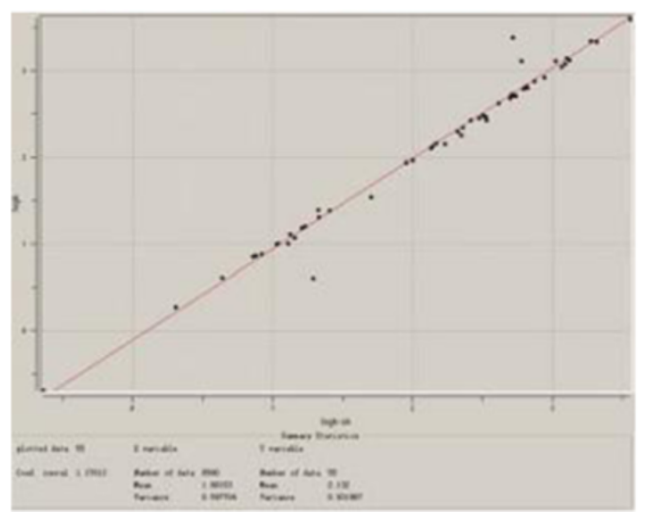

Figure 8 Permeability: a) simple kriging; b) simple kriging variance; c) coefficient of variation.

conditioning data is undersampled with respect to the rest of the grid. The ordinary cokriging variance map is shown in Figure 11b, which is more smooth than that of the simple cokriging variance map. The blue area that represents small variance tends to be wider, which stands for a better estimation result compared to simple cokriging. The estimation variance is small in gridblocks close to the conditioning data, and it becomes large in area far from the data. Near the conditioning data, the kriging variance becomes the nugget effect of the variogram.
The histogram, for the ordinary cokriging estimates, is shown in figure. The mean is 1.79 , the median is 1.84 , both of which are a bit smaller than the conditioning data. The standard deviation of ordinary cokriging estimates is 0.887; while for conditioning data, it is 0.941 . So it is likely that has a narrower spread than the conditioning data. In general, ordinary cokriging tends to gather the data to the center, making the data follow normal distribution. That is, most of the data distributes close to the mean value. 92 points are compared and 
a)

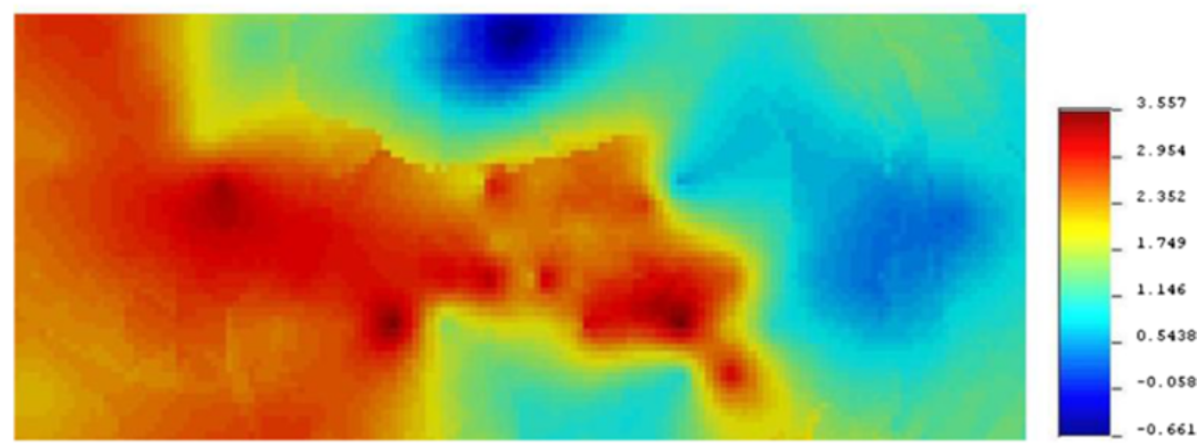

b)

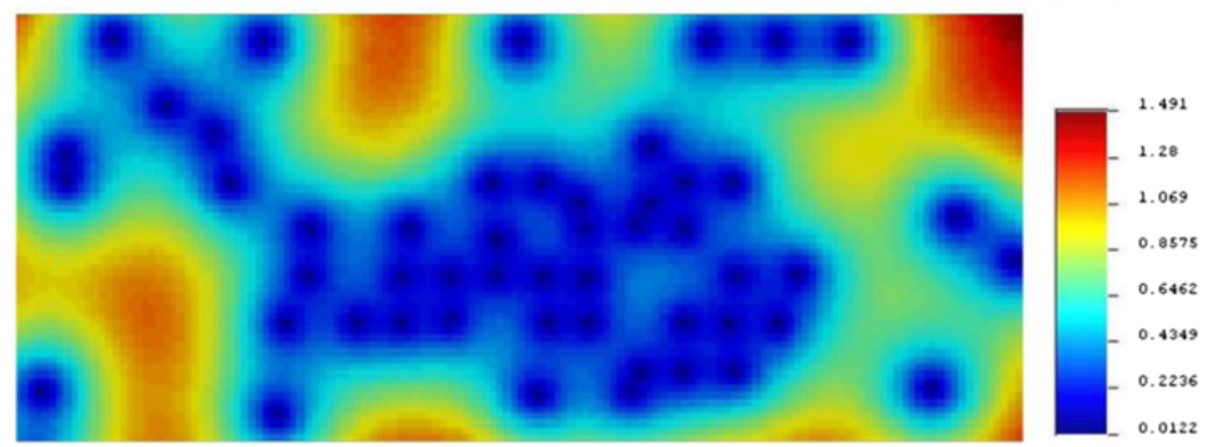

Figure 9 Permeability: a) ordinary kriging; b) ordinary kriging variance.

plotted in the scatter plot of gross log $\mathrm{K}$ data and ordinary cokriging estimation data. Ordinary cokriging provides good estimates even for values that are much larger than the mean of the data (1.79 to 2.13). All the plotted points fall approximately along the straight line, indicating high accuracy of the estimates.

\section{MM2 models}

Figure 12a shows the gross thickness map generated with MM2 cokriging. Overall, the map has a more smooth appearance. Transition areas exist in the estimate map, which represent gradual change among the subareas. The estimated distribution matches the original distribution well. The yellow and red areas represent the areas of which the permeability is relatively higher, while the blue areas stand for where the permeability is relatively lower. The good spatial continuity corresponds to the principal direction of the variogram. The small areas in the corner and bound of the map, is a result from the configuration of the conditioning data and the search neighborhood. In the northwest corner, the conditioning data is undersampled with respect to the rest of the grid.

The MM2 cokriging variance map is shown in Figure 12b, which is smoother than that of the Co-simple kriging variance map. The blue area that represents small variance tends to be wider. The estimation variance is small in gridblocks close to the conditioning data, and it becomes large in area far from the data. Near the conditioning data, the kriging variance becomes the nugget effect of the variogram. The histogram, for the MM2 cokriging estimates, is shown in figure. The mean is 1.86 , the median is 1.99 , both of which are a bit smaller than the conditioning data. The standard deviation of MM2 kriging estimates is 0.879 ; while for conditioning data, it is 0.941 . So it is likely that has a narrower spread than the conditioning data. 92 points are compared and plotted in the scatter plot of gross $\log \mathrm{K}$ data and MM2 estimation data. All the plotted points fall approximately along the straight line, indicating high accuracy of the estimates.

\section{Thickness SK vs OK}

To have a thorough understanding of the results, the difference analysis among the three methods is required. The difference in the estimation results from the two methods is shown in Figure 13. We can see that the map is, in general, covered with light orange (less than 0.3 from color bar), indicating the slight difference between the two methods. The estimate mean of OK is a bit larger than that of SK. In the west-north region, the light blue color implies the estimate of OK mean in this 




b)

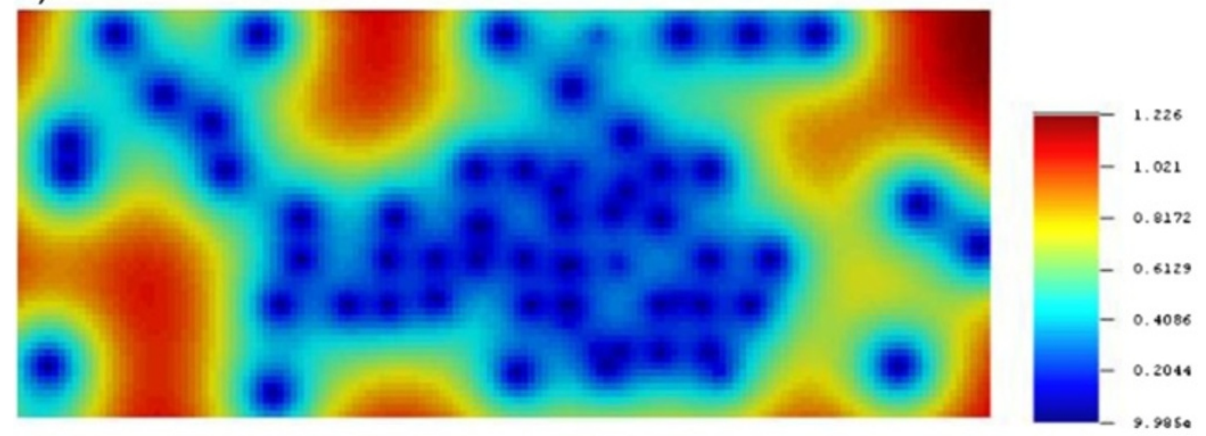

c)

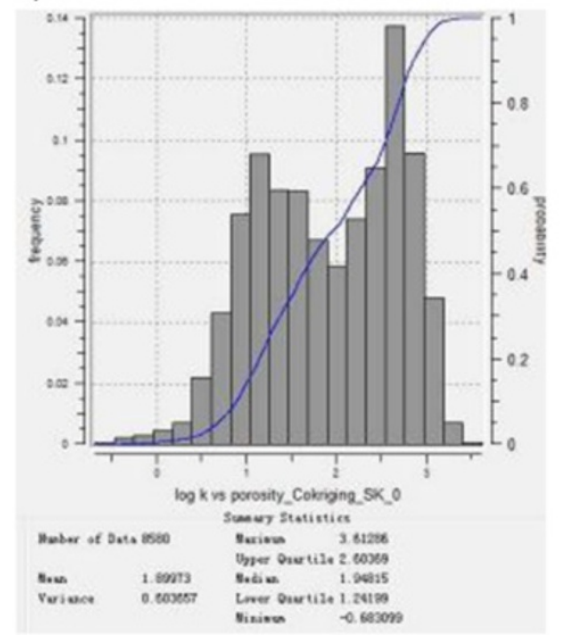

d)

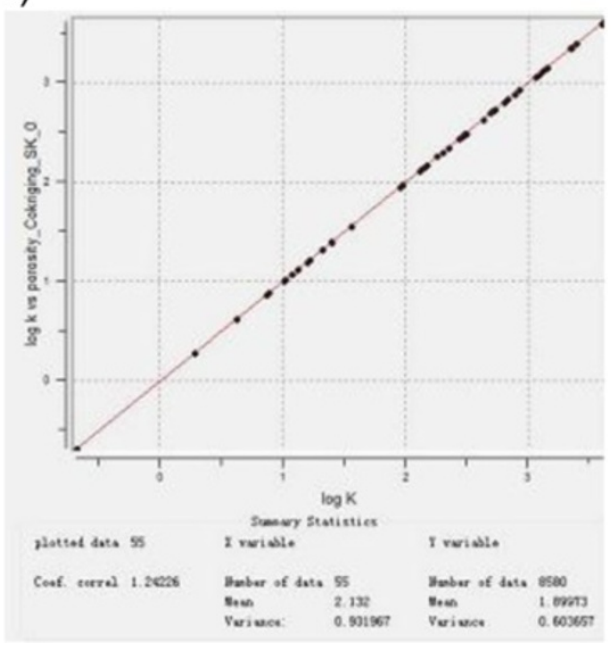

Figure 10 Permeability: a) simple cokriging; b) simple cokriging variance; c) histogram for simple cokriging; d) compare with true and simple cokriging value.

region is less than that of SK. This maybe mainly because this region is undersampled and there is less data available.

\section{Porosity}

\section{Simple kriging estimation vs. Ordinary kriging estiamtion}

The difference in the estimation results from the two methods is shown in Figure 14a. We can see that the map is, in general, covered with light blue, which implies the less difference between the two methods. After checking the sample data, we can find out that the light blue regions are where the true values exist. It thus well explains why there is less difference between the two methods. However, at where sample data are not available, the estimation difference between the two methods is remarkable. For example, in the north-east corner, the estimated values from simple kriging are significantly higher than that of ordinary kriging. In comparison, the estimated values, in the south-west region, are slightly lower than the values obtained via ordinary kriging. The 




b)

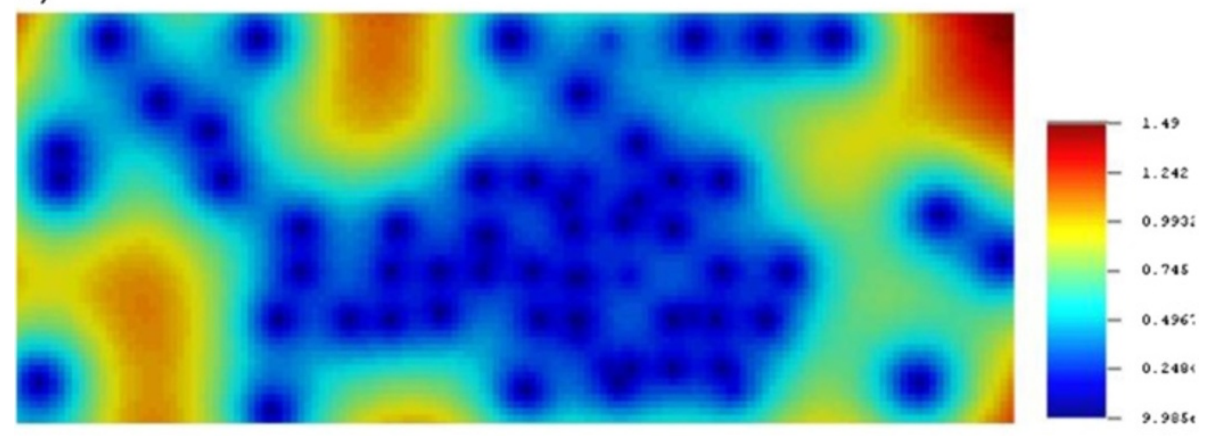

c)

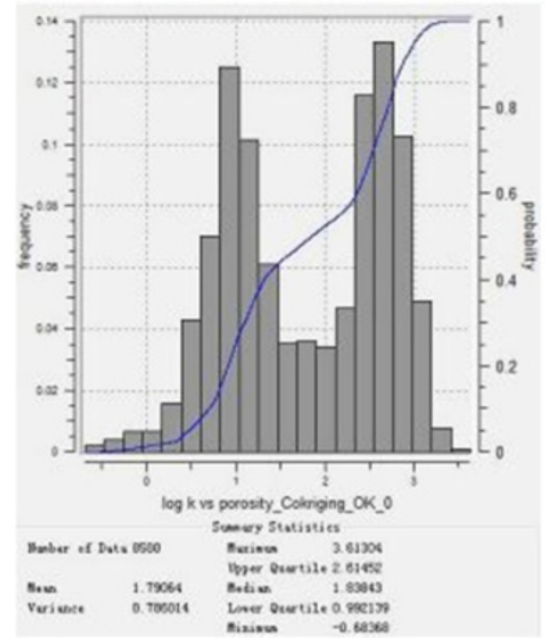

d)

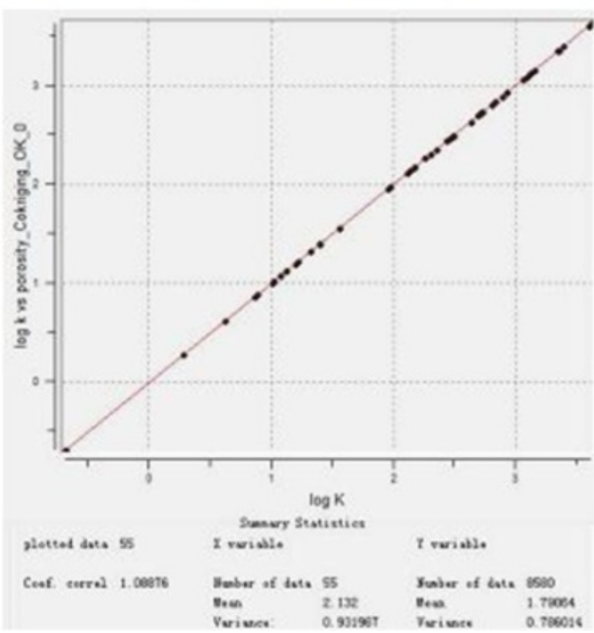

Figure 11 Permeability: a) ordinary cokriging; b) ordinary cokriging variance; c) histogram for ordinary cokriging; d) compare with logK and ordinary cokriging value.

difference in the estimation variance of the two methods is shown in Figure 14b. In general, the difference is inconsiderable. However, in the north-east corner, simple kriging estimates' variance is remarkably lower than the variance from ordinary kriging estimation.

\section{Simple \& ordinary kriging estimation vs. Simulation}

The two sets of estimation results are compared with the simulation results, as shown in Figure 15. Overall, the two maps are quite similar to each other. We can see that, in the north region, estimation results are lower than the simulation results. However, in the south region as well as the north-east corner, the estimation results are much larger than the simulation results.

\section{Permeability}

\section{SK vs OK}

The difference in the estimation results from the two methods is shown in Figure 16a. We can see that the map is, in general, covered with light blue, which implies 
a)

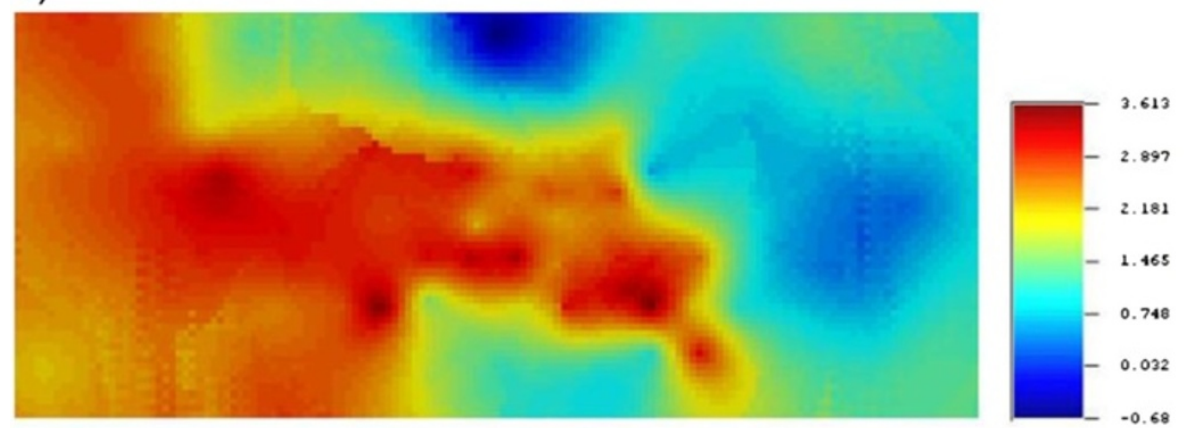

b)

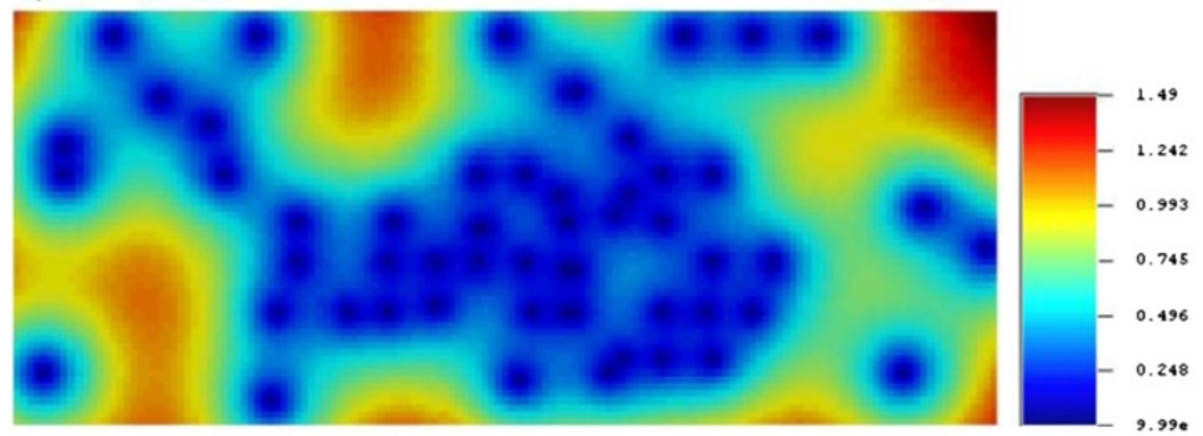

c)

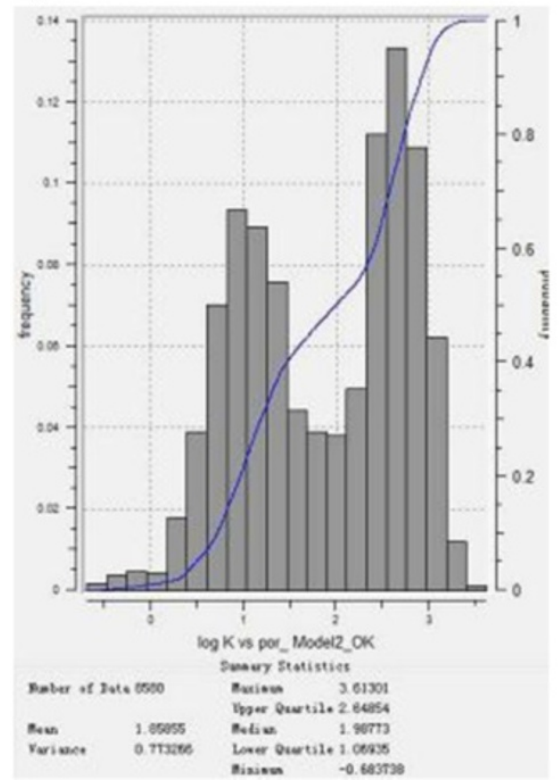

d)



Figure 12 Permeability: a) MM2 cokriging; b) MM2 cokriging variance; c) histogram for MM2 cokriging; d) compare with logK and MM2 estimation data.

the less difference between the two methods. After checking the sample data, we can find out that the light blue regions are where the true values exist. However, at where sample data are not available, the estimation difference between the two methods is remarkable. In the northeast corner, the estimated values from simple kriging are significantly higher than that of ordinary kriging.

\section{Simple cokriging vs ordinary cokriging}

The difference in the estimation of the two methods is shown in Figure 16b. In general, the difference is inconsiderable. However, in the northeast, southwest and southeast corner, simple kriging estimate is remarkably higher than the that of ordinary kriging estimation, where sample data is undersampled. 




Figure 13 Difference analysis for thickness SK vs. OK.

\section{OK vs ordinary cokriging}

The difference in the estimation results from the two methods is shown in Figure 16c. The map is, in general, saturated with light blue, which implies less difference between the two methods. However, in the places where the color is dark blue and red, the estimation difference between these two methods are remarkable. This happens mainly because there is no enough data collected in these regions.

\section{Ordinary cokriging vs MM2}

The difference in the estimation results from the two methods is shown in Figure 16d. We can see that the map is, in general, covered with orange (with the value of 0.05 from colorbar), indicating the slight difference between the two methods. And the estimate mean of ordinary kriging is a bit larger than that of MM2. Dark blue only exists in a few regions. These are where the estimate of MM2 is remarkably large than ordinary cokriging. This happens because there is not enough data collected.

\section{Thickness comparison between SK and OK}

a. The scatter plot of the simple kringing estimate and the ordinary kriging estimate is shown in Figure 17. The correlation coefficient is 0.973 , indicating a high similarity



b)

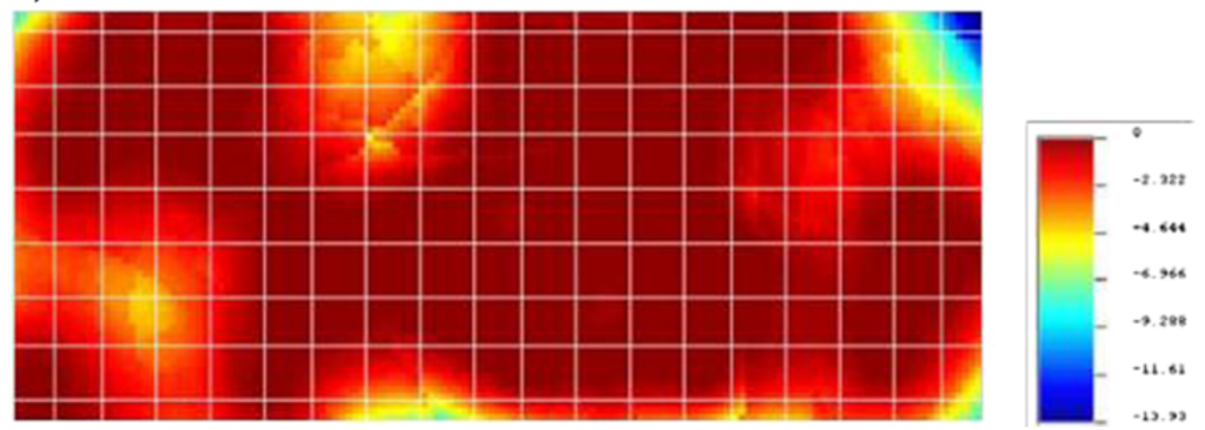

Figure 14 Porosity: a) difference in the estimation of SK and OK; b) difference in the estimation variance of SK and OK. 


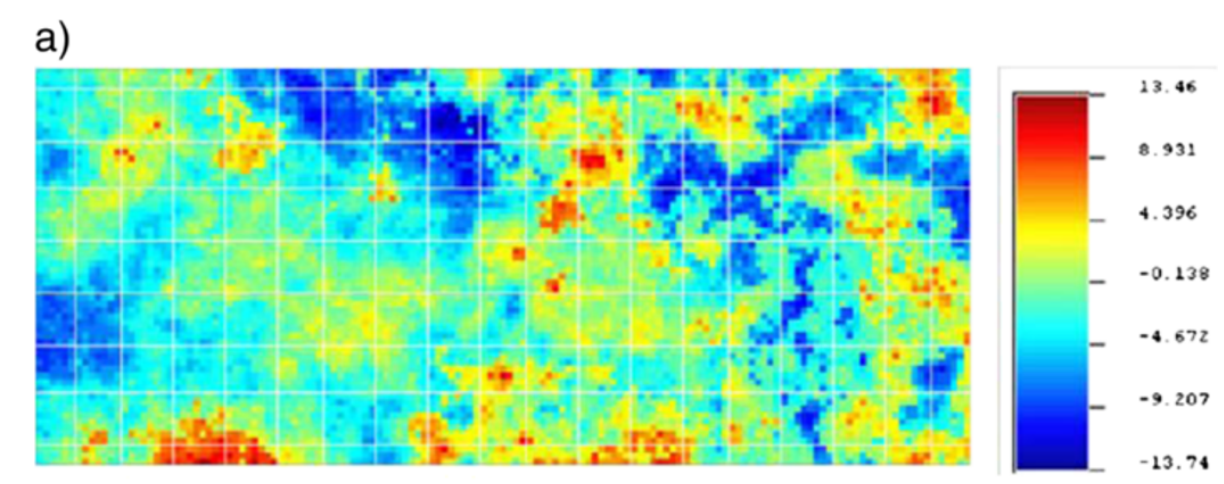

b)

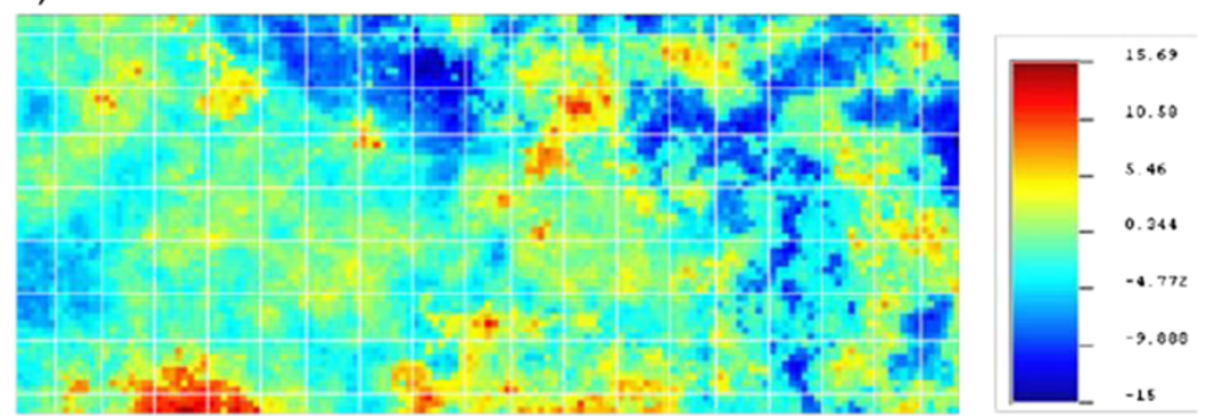

Figure 15 Porosity: simple (a) \& ordinary kriging (b) estimation results are compared with the simulation results.

between these two estimates. Most differences occur for gross thickness between 4 and $7 \mathrm{ft}$. And the ordinary kriging estimate tends to be a little larger than the simple kriging estimate. $b$. The scatter plot of the simple kringing variance and the ordinary kriging variance is shown in Figure 17. Ordinary kriging tends overestimate thickness. Figure 17 shows that the simple kriging variance is smaller than the error variance estimated with the ordinary kriging. In view of the estimation mean and variance, although SK estimate tends to be more close to the conditioning data for this case, OK and SK both can generate good estimation result.

\section{Porosity comparison between SK and OK}

a. The scatter plot of the simple kringing estimate and the ordinary kriging estimate is shown in Figure 18. The correlation coefficient is 0.984 , indicating a high similarity between these two estimates. Most differences occur for gross thickness between 12 and 19. The ordinary kriging estimate tends to be a little larger than the simple kriging estimate. $\mathrm{b}$. The scatter plot of the simple kringing variance and the ordinary kriging variance is shown in Figure 18. The simple kriging variance is only slightly smaller than the error variance estimated with the ordinary kriging.

\section{Log K comparison between SK and OK}

a. The scatter plot of the simple kringing estimate and the ordinary kriging estimate is shown in Figure 19. The correlation coefficient is 0.982 , indicating a high similarity between these two estimates. Most differences occur for gross thickness between 0.5 and 1 . And the ordinary kriging estimate tends to be a little larger than the simple kriging estimate. b. The scatter plot of the simple kringing variance and the ordinary kriging variance is shown in Figure 19. The correlation coefficient is 0.998 , indicating there is almost no difference between the estimation variance of the two.

\section{Log K comparison between simple cokriging and ordinary cokriging}

a. The scatter plot of the simple cokringing estimate and the ordinary cokriging estimate is shown in Figure 20. The correlation coefficient is 0.972 , indicating a high similarity between these two estimates. Most differences occur for $\log \mathrm{K}$ between 1 and 3 . And the ordinary cokriging estimate tends to be a little larger than the simple cokriging estimate. b. The scatter plot of the simple cokringing variance and the ordinary cokriging variance is shown in Figure 20. Figure 20 shows that the simple kriging variance is smaller than the error variance estimated with the ordinary kriging. In view of the estimation mean and variance, although simple kriging estimate tends to be more close to the conditioning data for this case, ordinary and simple kriging both can generate good estimation result. 
a)

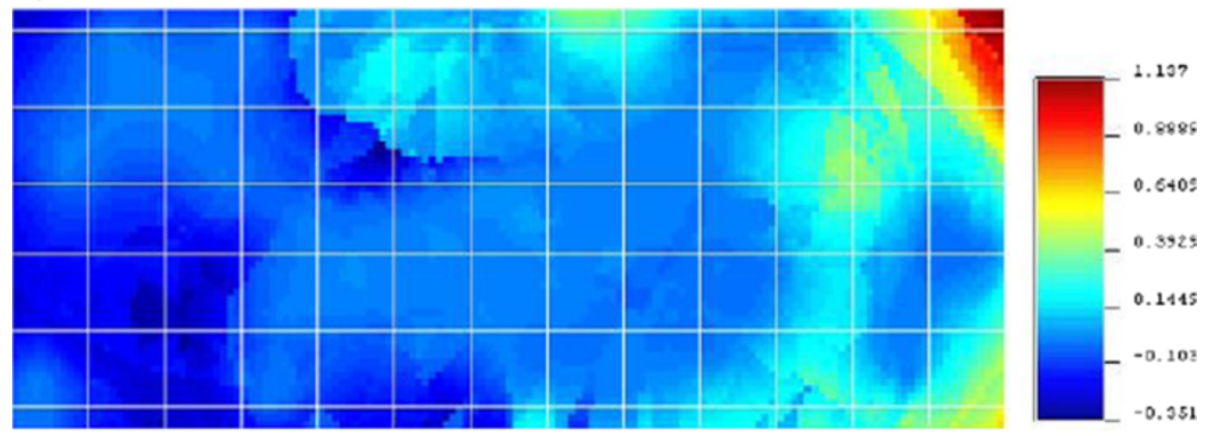

b)

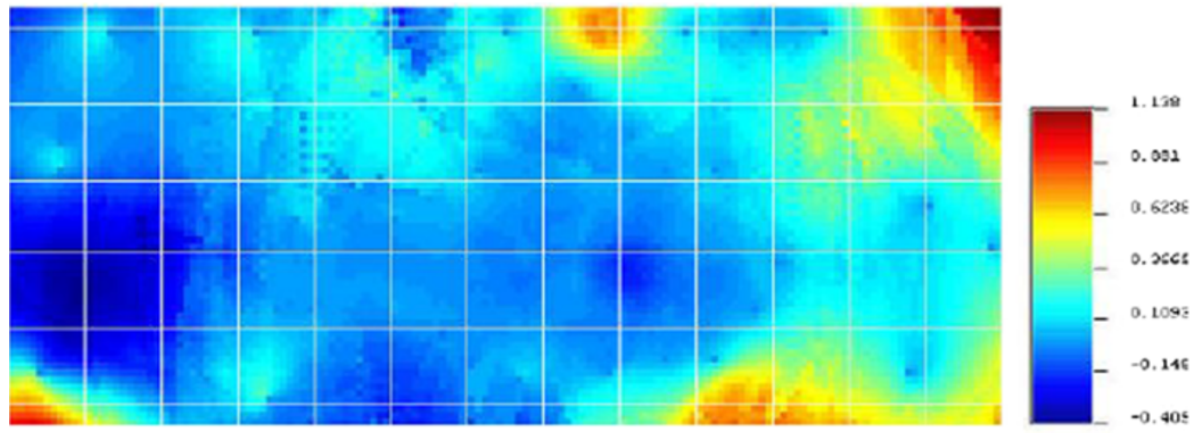

c)



d)



Figure 16 Permeability: a) difference in the estimation of SK and OK of $\log k$; b) difference in the estimation of simple cokriging and ordinary cokriging of $\log k$; c) difference in the estimation of OK and ordinary cokriging of log $k$; d) difference in the estimation of ordinary cokriging and $\mathrm{MM} 2$ of $\log \mathrm{k}$. 
a)

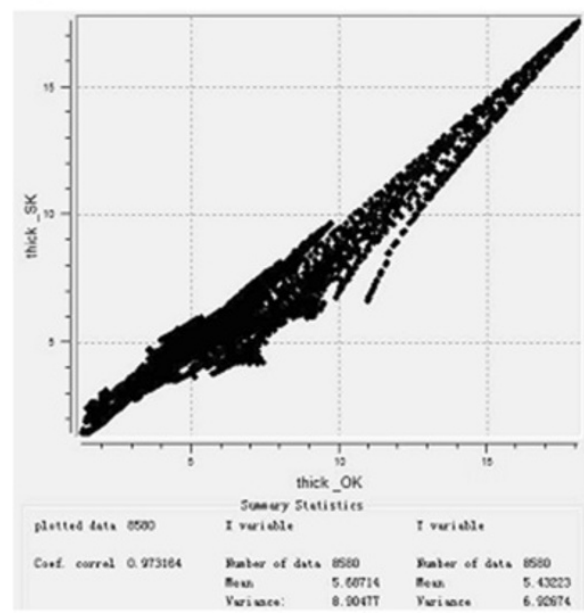

b)

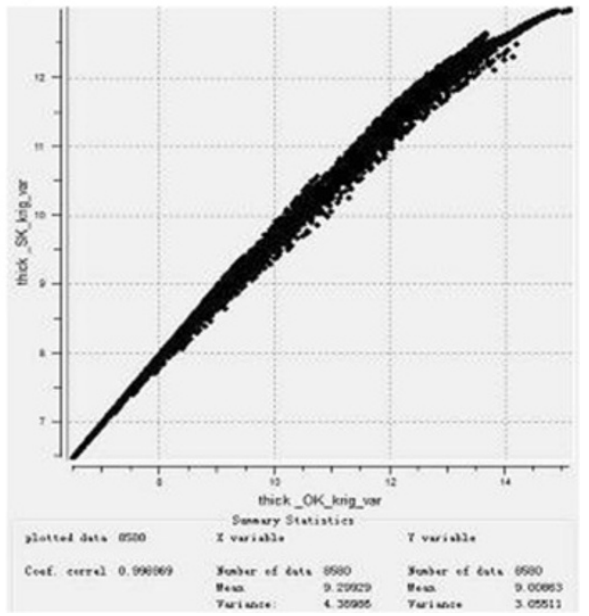

Figure 17 Thickness: a) the scatter plot of SK estimate and OK estimate for thickness; b) the scatter plot of SK variance and OK variance for thickness.

Log $\mathrm{K}$ comparison between ordinary kriging and ordinary cokriging

a. The spatial distributions of $\log \mathrm{K}$ are significantly different for ordinary cokriging and ordinary kriging methods. The map, generated with ordinary cokriging is not as smooth as ordinary kriging. The influence of porosity adds some unique features to the log $\mathrm{k}$ data map that ordinary kriging cannot capture with a single variogram model. The ordinary cokriging and ordinary kriging estimates differ significantly, as shown in Figure 21b. Ordinary kriging tends to overestimate $\log \mathrm{K}$. Figure $21 \mathrm{~b}$ shows that the cokriging variance was smaller than the error variance estimated with the ordinary kriging. This shows that additional information used in cokriging reduces the error variance in estimates.

\section{Log $\mathrm{K}$ comparison between ordinary cokriging and MM2} a. The spatial distributions of $\log \mathrm{K}$ are significantly different for MM2 and ordinary cokriging methods. From the scatter plot of both, the MM2 estimates tend to be larger than the ordinary full cokriging estimates, as shown in Figure 22b. MM2 cokriging tends overestimate log K.



b)

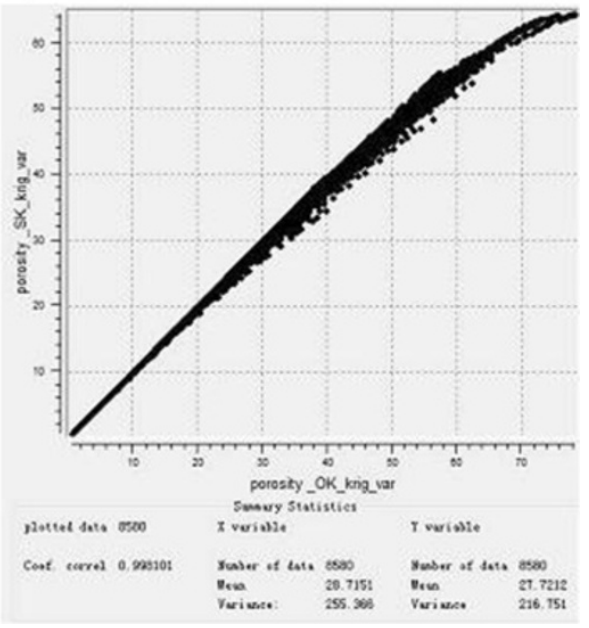

Figure 18 Porosity: a) The scatter plot of SK estimate and OK estimate for porosity; b) the scatter plot of SK variance and OK variance for porosity. 


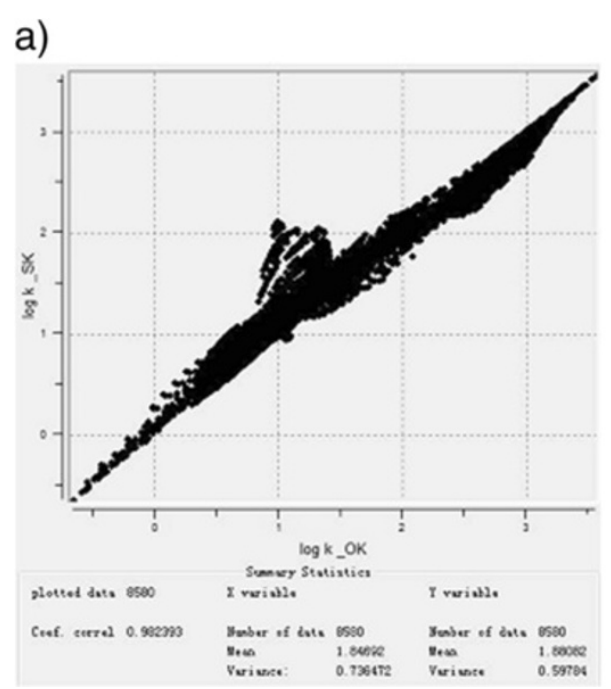

b)

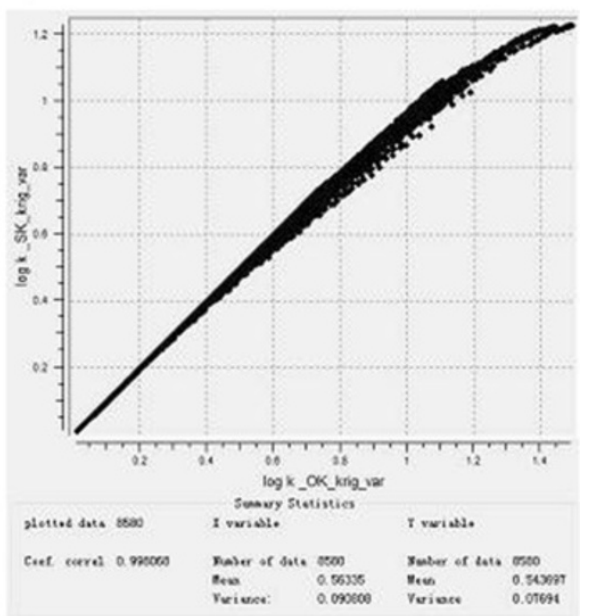

Figure 19 Permeability: a) the scatter plot of SK estimate and OK estimate for permeability; b) The scatter plot of SK variance and OK variance for permeability.

Figure 22b shows that the full cokriging variance is smaller than the error variance estimated with the MM2. This shows that additional information used in ordinary cokriging reduces the error variance in estimates.

\section{Conclusions}

Log transformation of the permeability can be applied to overcome the problem that the permeability data are clustered at the lower end of the study region. This would minimize the effect of the extreme and order-of-magnitude variations within the data points, and result a better identification of the spatial structure.
From an estimation point of view, it is always preferable to start with an isotropic variogram structure before the investigation of anisotropy. From studying the case of flow unit 5, a conclusion is made that anisotropic variogram could achieve better spatial structure and capture more interpretable spatial relationship than isotropic variogram.

Generally, the estimation results of the ordinary kriging and simple kriging method are similar. The correlation coefficient between the two estimates is quite close to 1 for thickness, porosity and $\log \mathrm{K}$. However, the variance maps of the estimates obtained via ordinary kriging are much smoother than that via simple kriging. Transition a)

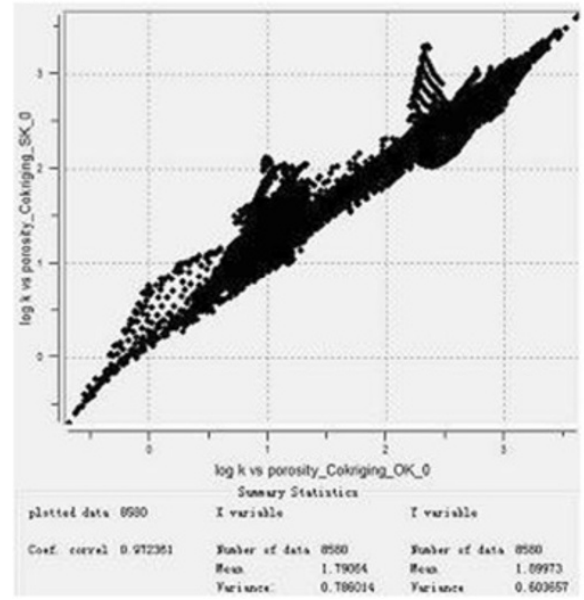

b)

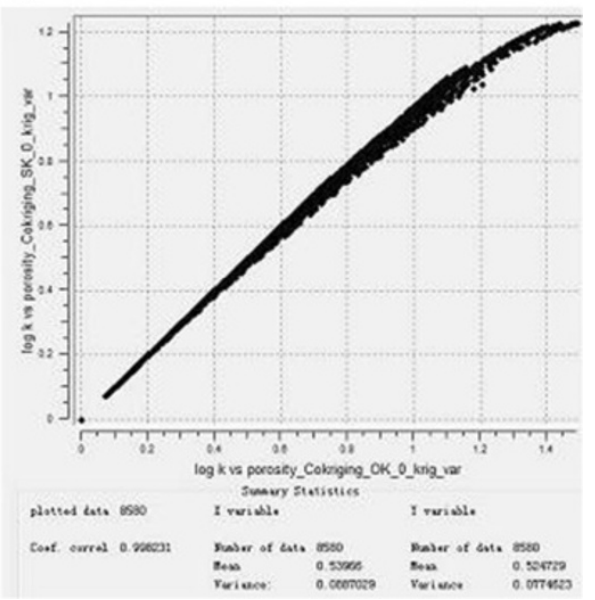

Figure 20 Permeability: a) the scatter plot of simple cokriging and ordinary cokriging for permeability; b) the scatter plot of simple cokriging variance and ordinary cokriging variance for permeability. 
a)

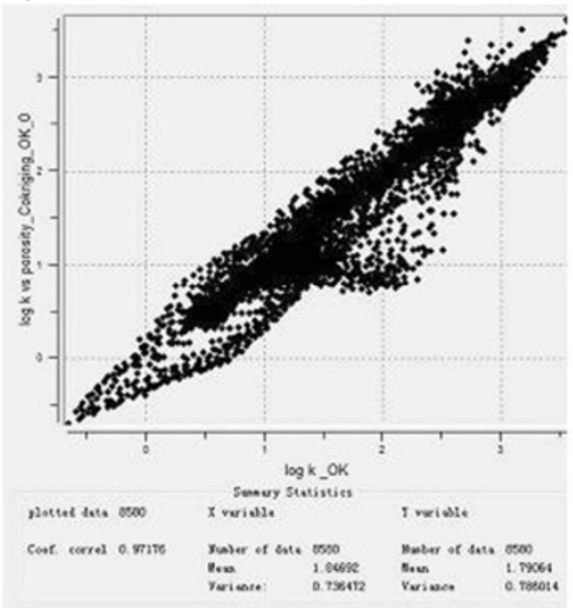

b)



Figure 21 Permeability: a) the scatter plot of OK and ordinary cokriging for permeability; b) the scatter plot of OK variance and ordinary cokriging variance for permeability.

areas exist in ordinary kriging map, showing more gradual change among the subareas. This is because the sample mean value has been ignored in ordinary kriging estimation.

The estimation methods such as simple kriging and ordinary kriging tend to eliminate the extreme values observed in the sample data, and narrow the spread of the data distribution. Consequently, these two methods appear to gather the data to the center and make the data follow normal distribution. This is the reason why most of the data distribute close to the mean value.

Compared with conventional estimation techniques, the simulation method could well reflect the reservoir's intrinsical characteristics in terms of the associated extreme values. From the case study of the porosity, we can see that both the estimation and simulation methods could obtain the similar results. There is almost no any remarkable difference at where the sample data are sufficient. However, at the unsampled locations, the estimation results are much larger than the simulation results. For example, the south region as well as the north-east corner.

Cokriging estimation employs the secondary variable to estimate the first variable. The two variables should be linearly related and have a strong relationship with each other. Unlike ordinary kriging, in which we only use the values surrounding the sampled locations to estimate the unsampled locations, Cokriging method could
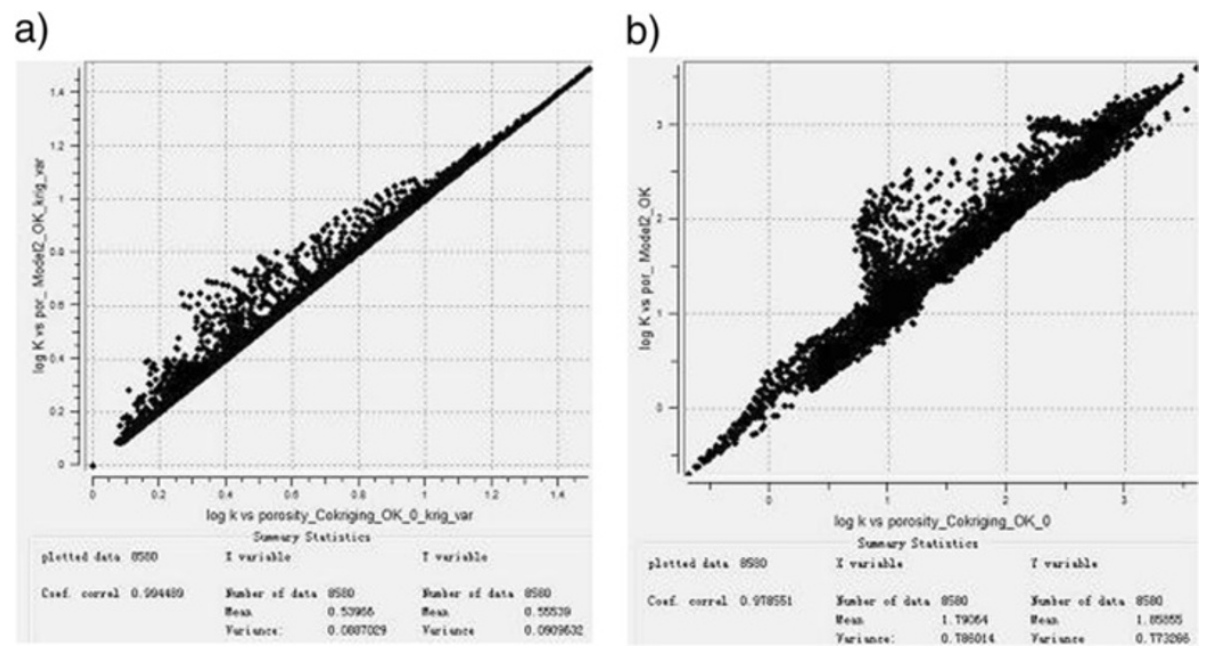

Figure 22 a) The scatter plot of ordianary cokriging and MM2 for permeability; b) the scatter plot of ordianary cokriging variance and MM2 for permeability. 
be able to improve the estimation and reduce the uncertainty of the estimation with the assist of the spatial information available from the secondary variable.

Furthermore, employing porosity as the secondary variable would add some unique features to the estimation of $\log \mathrm{K}$, and these features cannot be captured by either of the simple kriging and ordinary kriging methods via a single variogram model. In addition, Cokriging would tend to reduce the error variance compared with the simple and ordinary kriging methods.

For thickness, both the ordinary kriging and the simple kriging methods could achieve better estimation results than others. For porosity, the simple kriging, the ordinary kriging and the Sigsm methods could generate similar estimation results. For permeability, there is no much difference between the results of simple cokriging and ordinary cokriging methods. However, ordinary cokriging method could produce better results than both of the ordinary kriging and the MM2 methods.

Cross validation can be used to select the optimum kriging parameters, which can be utilized further in the kriging methods if it is permitted. The cross-validation is verified by the existence of strong relationship between the estimate and gross data, as well as the small correlations between the estimated values and their errors. However, it is a trial and error procedure and time consuming job.

\section{Methods}

Once initial data sets are prepared, quality controlled, and loaded into the geostatistical software (like SGeMS), a typical work flow, with iterations, might be: (1) data mining; (2) spatial continuity analysis and modeling; (3) search ellipse design; (4) model crossvalidation; (5) kriging; (6) conditional simulation; (7) model uncertainty assessment.

\section{Data mining}

An early and fundamental step in any science starts at the descriptive stage. Until facts are accurately gathered and described, an analysis of their causes is premature. Because statistics generally deals with quantities of data, not with a single datum, we need some means to deal with the data in a manageable form. Thus, much of statistics deals with ways of describing the data and understanding relationships between pairs of variables. Data speak most clearly when they are organized (Isaaks and Srivastava 1989).

Because there is no one set of prescribed steps in data mining, you should follow your instincts in explaining anomalies in the data set. By using various tools, you gain clearer understanding of your data and also discover possible sources of errors. Errors are easily overlooked, especially in large data sets and when computers are involved, because we simply become detached from our data. Thorough analysis fosters an intimate understanding of the data that can flag spurious results.

Classical statistical data analysis includes data posting, computation of means and variances, making scatterplots to investigate the relationship between two variables, and identification of subpopulations and potential outliers.

Histograms, graphical representations of the data distribution of a single variable, record how often values fall within specified intervals or classes. A bar depicts each class, and its height is proportional to the number of values within that class. The histogram shape informs us about the distribution of the data values. Ideally, we like to see a bell-shaped, symmetrical distribution around the mean value. This is referred to as a normal, or Gaussian, distribution and has a predictable shape based on the data mean and variance. Many statistical and geostatistical methods assume such a data model. If the shape is skewed to either side of the mean, then often it is necessary to adjust the shape by transforming the data into Gaussian form. Complex histograms may indicate mixing of multiple distributions. Categorization of the data (e.g., by facies) often identifies the underlying distributions.

\section{Spatial continuity analysis and modeling}

Variables of interest in the petroleum industry (e.g., porosity, permeability, saturation, sand/shale volumes, etc.) are the product of a vast number of complex physical and chemical processes. These processes superimpose a spatial pattern on reservoir rock properties, and it is important to understand the scales and directional aspects of these features for efficient hydrocarbon production. The spatial component makes these variables complicated, and we are forced to admit uncertainty about their distribution between wells. Because deterministic models do not handle uncertainties associated with these variables, a geostatistical approach is used because its foundation is probabilistic theory (covariance models) that recognizes these inevitable uncertainties.

\section{The spatial model}

Spatial continuity analysis quantifies the variability of sample properties with respect to distance and direction (geographic location is considered only if the data exhibit a trend, a property known as nonstationarity).

Quantifying spatial information involves comparing data values at one location with values of the same attribute at other locations. For example, two wells in close proximity are more likely to have similar reservoir properties than two wells farther apart. The key questionwhat we want to know-is what measured values tell us about reservoir properties at unsampled locations. 


\section{Geometric Anisotropy}

Geometric Anisotropy typically is observed when the variograms in the directions of maximum and minimum continuity show a similar shape and sill but different range. The range in the direction of maximum continuity, $\mathrm{u}$, is $\mathrm{u}$ a, while the range in the direction of minimum continuity is $\mathrm{v}$ a. We assume that the two directions are perpendicular to each other.

To model the two variograms with the same sill, we have to use the same combination of linear models in both directions expect with different ranges. For example, with a linear combination of nugget and spherical models, the model in the $\mathrm{u}$ direction is

$$
\gamma_{u}(L)=C_{0}+C_{1} M_{S a_{u}}(L) \text {. }
$$

And the model in the $\mathrm{v}$

$$
\gamma_{v}(L)=C_{0}+C_{1} M_{S a_{v}}(L) .
$$

\section{Modeling of Cross Variograms}

Cross Variograms can be modeled with the same models used to model variograms. As before, the rule of the use of a minimum number of parameters to model the variogram applies, and the rule of the condition of positive definiteness should be satisfied.

To satisfy the condition of positive definiteness, certain additional restrictions are imposed when modeling the cross variogram. If $\mathrm{x}$ and $\mathrm{y}$ are considered as two variables, the variograms for the two variables $\mathrm{x}$ and $\mathrm{y}$ are modeled, respectively, as

$$
\begin{aligned}
& \gamma_{x}(L)=C_{0 x}+C_{1 x} M_{S a}(L) \\
& \gamma_{v}(L)=C_{0 v}+C_{1 v} M_{S a}(L) .
\end{aligned}
$$

Note that both variograms need to be modeled with the same linear combination of structures and that the range for both variograms must be the same. The only difference between the two structures are sill values, which can be different. To model the cross variogram between the two variables, we can write

$$
\gamma_{C x v}(L)=C_{0 x v}+C_{1 x v} M_{S a}(L) .
$$

As in modeling variograms, we are restricted by the same linear combinations of models in modeling the cross variogram, and the range must also be the same for the cross-variogram structure. In addition, the coefficients of the model should be defined so that the following two conditions are satisfied.

$$
\begin{aligned}
& C_{0 x} C_{0 y}>C_{0 x y}^{2} \\
& C_{1 x} C_{1 y}>C_{1 x y}^{2} .
\end{aligned}
$$

That is, for a given model, the product of the coefficients of individual variable variogram models should be greater than the square of the coefficient of the cross variogram.

\section{Search ellipse design}

Because computers are used in mapping, we must instruct the program how to gather and use control points during interpolation. Most familiar with computer mapping know that this involves designing a search ellipse or neighborhood. We must specify the length of the search radius, the number of sectors (typically four or eight), and the number of data points per sector. Most common mapping programs allow the user to specify only one radius; thus, the search ellipse is circular (isotropic). However, during geostatistical analysis, we often find that the spatial model is anisotropic. Thus, we should design the search ellipse based on the spatial model correlation scales, aligning the search ellipse azimuth with the major axis of anisotropy.

Kriging is a geostatistical interpolation technique. It is a linear weighted-averaging method, similar to inverse weighted distance. However, kriging weights depend on a model of spatial correlation. Therefore, it is possible to create a map exhibiting strong anisotropy, resulting in a map that "looks" more geologically plausible.

Depending on the specific application, different procedures have been used for the purpose of estimation. In this study, we will introduce the Simple Kriging (CK), Ordinary Kriging $(\mathrm{OK})$ and Cokriging with a case study in the following chapters. Here we will briefly recall the definition of these algorithms.

\section{Simple kriging}

Simple kriging requires a knowledge of population mean, which may not be known in practice because without prior assumptions. Therefore, this type of kriging procedure is not quite popular.

\section{Ordinary kriging}

Ordinary kriging is the most popular techbique, which eliminates the need for knowledge of mean value. It also is easier to adapt to local variations. It is, without question, is the most widely applied kriging technique.

\section{Cokriging}

The Cokriging algorithm integrates the information carried by a secondary variable related to the primary attribute being estimated. The kriging system of equations is then extended to take into account that extra information. The Markov Model 1 (MM1) and the Markov Model 2 (MM2). The Markov models ( MM1 or MM2) can only be solved with simple cokriging; using ordinary cokriging would lead to ignoring the secondary variable since the 
sum of weights for the secondary variable must be equal to zero.

Kriging is a deterministic method that has a unique solution offering the best estimate. It does not pretend to represent the actual variability of the studied attribute. It can be used in the traditional way that other mathematical interpolation methods have been used. It has the added value of incorporating the spatial model and thus more reliably depicting the shapes of geologic features.

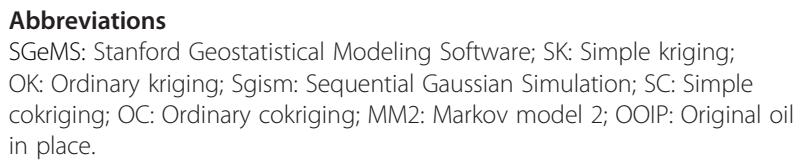

\section{Competing interests}

- In the past five years have you received reimbursements, fees, funding, or salary from an organization that may in any way gain or lose financially from the publication of this manuscript, either now or in the future? Is such an organization financing this manuscript (including the article-processing charge)? If so, please specify.

$\rightarrow \mathrm{NO}$.

- Do you hold any stocks or shares in an organization that may in any way gain or lose financially from the publication of this manuscript, either now or in the future? If so, please specify.

$\rightarrow \mathrm{NO}$.

- Do you hold or are you currently applying for any patents relating to the content of the manuscript? Have you received reimbursements, fees, funding, or salary from an organization that holds or has applied for patents relating to the content of the manuscript? If so, please specify. $\rightarrow \mathrm{NO}$.

- Do you have any other financial competing interests? If so, please specify. $\rightarrow \mathrm{NO}$.

The authors declare that they have no competing interest.

- Are there any non-financial competing interests (political, personal, religious, ideological, academic, intellectual, commercial or any other) to declare in relation to this manuscript? If so, please specify. $\rightarrow \mathrm{NO}$.

\section{Authors' contributions}

$\mathrm{ZS}, \mathrm{ZY}$, and WM made substantial contributions to conception and design, or acquisition of data, or analysis and interpretation of data. ZS brought up the innovation and designed the study. ZY proposed the methodology and wrote the code. WM collected the data and edited the format. XX and CF were involved in drafting the manuscript and revised it critically for important intellectual content. All authors gave final approval of the version to be published. All authors read and approved the final manuscript.

\section{Acknowledgements}

This study was part of a research project funded by Petroleum Technology Research Centre, Canada. We also feel grateful for the support from Faculty of Engineering and Applied Science, University of Regina.

Received: 13 December 2013 Accepted: 6 January 2014

Published: 18 January 2014

\section{References}

Abdideh M, Bargahi D (2012) Designing a 3D model for the prediction of the top of formation in oil fields using geostatistical methods. Geocarto Int 27(7):569-579

Abdideh M, Mahmoudi N (2013) UCS prediction: a new set of concepts for Reservoir Geomechanical Modeling. Pet Sci Technol 31(24):2629-2635

Bueno JF, Drummond RD, Vidal AC, Sancevero SS (2011) Constraining uncertainty in volumetric estimation: A case study from Namorado Field, Brazil. J Pet Sci Eng 77(2):200-208

Chen Y, Park K, Durlofsky L (2011) Statistical assignment of upscaled flow functions for an ensemble of geological models. Comput Geosci 15(1):35-51
Cressie N, Hawkins D (1980) Robust estimation of the variogram: I. Math Geol 12(2):115-125

Esmaeilzadeh S, Afshari A, Motafakkerfard R (2013) Integrating artificial neural networks technique and geostatistical approaches for 3D Geological Reservoir Porosity Modeling with an example from one of Iran's oil fields. Pet Sci Technol 31(11):1175-1187

Fegh A, Riahi M, Norouzi G (2013) Permeability prediction and construction of 3D geological model: application of neural networks and stochastic approaches in an Iranian gas reservoir. Neural Comput \& Applic 23(6):1763-1770

Geoff B (2007) S-GeMS Tutorial Notes, Kansas Geological Survey

Habibnia B, Momeni A (2012) Reservoir characterization in Balal oil field by means of inversion, attribute, and geostatistical analysis methods. Pet Sci Technol 30(15):1609-1618

Huysmans M, Dassargues A (2013) The effect of heterogeneity of diffusion parameters on chloride transport in low-permeability argillites. Environ Earth Sci 68(7):1835-1848

Isaaks EH, Srivastava RM (1989) Applied Geostatistics. Oxford University Press, New York

Kelkar M, Perez G (2002) Applied Geostatistic for Reservoir Characterization Kelsall J, Wakefield J (2002) Modeling spatial variation in disease risk. J Am Stat Assoc 97(459):692-701

Malvić T, Jović G (2012) Thickness maps of neogene and quaternary sediments in the Kloštar Field (Sava Depression, Croatia). J Maps 8(3):260-266

Nava E, Pintos S, Queipo NV (2010) A geostatistical perspective for the surrogate-based integration of variable fidelity models. J Pet Sci Eng $71(1-2): 56-66$

Qi L, Carr TR, Goldstein RH (2007) Geostatistical three-dimensional modeling of oolite shoals, St. Louis Limestone, southwest Kansas. AAPG Bull 91(1):69-96

Remy N, Boucher A, Wu J (2009) Applied Geostatistics with SGeMS. Cambridge University Press, UK

Remy N (2004) Geostatistical Earth Modeling Software: User's Manual

Schmidt G, Schröder W (2011) Regionalisation of climate variability used for modelling the dispersal of genetically modified oil seed rape in Northern Germany. Ecol Indic 11(4):951-963

Soleymani H, Riahi MA (2012) Velocity based pore pressure prediction-A case study at one of the Iranian southwest oil fields. J Pet Sci Eng 94-95:40-46

Wilson CE, Aydin A, Durlofsky LJ, Boucher A, Brownlow DT (2011) Use of outcrop observations, geostatistical analysis, and flow simulation to investigate structural controls on secondary hydrocarbon migration in the Anacacho Limestone, Uvalde, Texas. AAPG Bull 95(7):1181-1206

Zarei A, Masihi M, Salahshoor K (2011) Comparison of different univariate and multivariate geostatistical methods by porosity modeling of an Iranian oil field. Pet Sci Technol 29(19):2061-2076

doi:10.1186/2193-2697-3-7

Cite this article as: Zhao et al:: Thickness, porosity, and permeability prediction: comparative studies and application of the geostatistical modeling in an Oil field. Environmental Systems Research 2014 3:7.

\section{Submit your manuscript to a SpringerOpen ${ }^{\odot}$ journal and benefit from:}

- Convenient online submission

- Rigorous peer review

- Immediate publication on acceptance

- Open access: articles freely available online

- High visibility within the field

- Retaining the copyright to your article

Submit your next manuscript at $>$ springeropen.com 